\title{
Lattice and order properties of the poset of regions in a hyperplane arrangement
}

\author{
NATHAN READING
}

ABSTRACT. We show that the poset of regions (with respect to a canonical base region) of a supersolvable hyperplane arrangement is a congruence normal lattice. Specifically, the poset of regions of a supersolvable arrangement of rank $k$ is obtained via a sequence of doublings from the poset of regions of a supersolvable arrangement of rank $k-1$. An explicit description of the doublings leads to a proof that the order dimension of the poset of regions (again with respect to a canonical base region) of a supersolvable hyperplane arrangement is equal to the rank of the arrangement. In particular, the order dimension of the weak order on a finite Coxeter group of type A or B is equal to the number of generators. The result for type A (the permutation lattice) was proven previously by Flath [11].

We show that the poset of regions of a simplicial arrangement is a semi-distributive lattice, using the previously known result [2] that it is a lattice. A lattice is congruence uniform (or "bounded" in the sense of McKenzie [18]) if and only if it is semi-distributive and congruence normal [7]. Caspard, Le Conte de Poly-Barbut and Morvan [4] showed that the weak order on a finite Coxeter group is congruence uniform. Inspired by the methods of [4], we characterize congruence normality of a lattice in terms of edge-labelings. This leads to a simple criterion to determine whether or not a given simplicial arrangement has a congruence uniform lattice of regions. In the case when the criterion is satisfied, we explicitly characterize the congruence lattice of the lattice of regions.

\section{Main results}

We begin by listing the main results, with most definitions put off until Section 2.

Theorem 1. The poset of regions (with respect to a canonical base region) of a supersolvable hyperplane arrangement is a congruence normal lattice.

A lattice is called congruence normal if it can be obtained from the one-element lattice by a sequence of doublings of convex sets [6]. The doublings in Theorem 1 correspond to adjoining hyperplanes one by one to form the arrangement. Björner,

Presented by R. Freese.

Received August 24, 2002; accepted in final form July 9, 2003.

2000 Mathematics Subject Classification: 06B05; 06A07, 20 F55.

Key words and phrases: Bounded lattice, Cayley lattice, congruence normal, congruence uniform, Coxeter group, critical pair, doubling, hyperplane arrangement, order dimension, order quotient, permutation lattice, poset of regions, semi-distributive lattice, simplicial, subcritical pair, supersolvable, weak order.

The author was partially supported by NSF grants DMS-9877047 and DMS-0202430. 
Edelman and Ziegler [2] showed that the poset of regions (with respect to a canonical base region) of a supersolvable hyperplane arrangement is a lattice. The proof given here of Theorem 1 provides a different proof of the lattice property, but uses several key observations made in [2].

The considerations used to prove Theorem 1 also lead to the following theorem:

Theorem 2. The order dimension of the poset of regions (with respect to a canonical base region) of a supersolvable hyperplane arrangement is equal to the rank of the arrangement.

In particular, the order dimension of the weak order (or Cayley lattice) on a Coxeter group of type A or B is the number of generators. The result for the permutation lattice (type A) was proven previously by Flath [11]. Theorem 2 does not apply to other Coxeter groups, as the Coxeter arrangements of other types are known not to be supersolvable [1].

The semi-distributive condition on a lattice is strictly weaker than the distributive condition, and is different from the notion of "meet-distributive" and "joindistributive" lattices found in the study of convex geometries [10].

Theorem 3. The poset of regions (with respect to any base region) of a simplicial hyperplane arrangement is a semi-distributive lattice.

Björner, Edelman and Ziegler [2] proved previously that the poset of regions of a simplicial arrangement is a lattice, and we do not give a new proof of that fact. Le Conte de Poly-Barbut [17] proved that the weak order on a finite Coxeter group is a semi-distributive lattice. Duquenne and Cherfouh [8] had previously proven that weak order on Coxeter groups of type $\mathrm{A}$ is a semi-distributive lattice. The proof given here of Theorem 3 incorporates elements of Le Conte de Poly-Barbut's proof and elements of the proof of the lattice property in [2].

A lattice that is both congruence normal and semi-distributive is called congruence uniform or bounded. This use of the term "bounded" conflicts with another usage of the term: a poset with a unique minimal element and a unique maximal element is often called bounded. Therefore, throughout this paper, we will use the term "congruence uniform" rather than "bounded." A lattice is congruence uniform if and only if it can be obtained from the one-element lattice by a sequence of doublings of intervals [6]. Caspard, Le Conte de Poly-Barbut and Morvan [4] proved that Cayley lattices (weak orders) of finite Coxeter groups are congruence uniform. The result for type A had been proven previously by Caspard [3]. The proof given in [4] introduces the idea of proving congruence uniformity by means of an edge-labeling. Conditions are given on an edge-labeling of a lattice, so that the existence of such a labeling is sufficient to prove that a semi-distributive lattice has the " $\mathcal{H H}$ property" and in particular is congruence uniform. 
In this paper, we take the edge-labeling idea of [4] in a different direction. We define the notion of a CN-labeling and prove the following (where $E(L)$ is the set of edges, or cover relations of $L)$ :

Theorem 4. A lattice $L$ is congruence normal if and only if there exists a $C N$ labeling $\gamma: E(L) \rightarrow P$ for some poset $P$.

The proof of the "if" part of the statement is similar to the proof given in [4] for $\mathcal{H H}$ lattices, and the proof of "only if" uses a characterization of congruence normality essentially due to Day [7]. As part of the proof of Theorem 4 we show that one can always take $P$ to be $\operatorname{Irr}(\operatorname{Con}(L))$, the poset of join-irreducibles of the congruence lattice of $L$. The usefulness of Theorem 4 is that it makes possible a non-inductive proof of the congruence normality of a class of posets. The induction is hidden in the proof of Theorem 4. Theorem 4 is not used to prove Theorem 1 , because in that case there is a direct inductive proof which does not require a separate proof that the poset of regions is a lattice, and which leads to Theorem 2 .

We apply Theorem 4 to simplicial arrangements to characterize which pairs $(\mathcal{A}, B)$ have a congruence uniform lattice of regions. One can decompose each hyperplane in $\mathcal{A}$ into pieces called shards and define a directed graph $\operatorname{Sh}(\mathcal{A}, B)$ such that for a simplicial arrangement $\mathcal{A}$, the lattice $\mathcal{P}(\mathcal{A}, B)$ is congruence uniform if and only if $\mathcal{S h}(\mathcal{A}, B)$ is acyclic (Theorem 25). In that case the transitive closure of $\operatorname{Sh}(\mathcal{A}, B)$ is isomorphic to the poset of join-irreducibles of the congruence lattice of $\mathcal{P}(\mathcal{A}, B)$. In [22], this fact is used to explicitly determine the poset of joinirreducibles of the congruence lattice of the weak order for several types of finite Coxeter groups.

We also define a smaller directed graph $\mathcal{Q}(\mathcal{A}, B)$ which, for a simplicial arrangement $\mathcal{A}$ is acyclic if and only if $\mathcal{A}$ has a quotient ordering (see Section 2). When $\mathcal{A}$ is a Coxeter arrangement, $\mathcal{Q}(\mathcal{A}, B)$ is acyclic and thus the weak order on a finite Coxeter group has a quotient ordering and in particular is a congruence uniform lattice [4].

Theorems 1, 2 and 3 can be proven in the greater generality of oriented matroids. The reader will be able to fill in the details using [2, Section 6] as a reference.

The remainder of this paper is organized as follows: Section 2 contains definitions and preliminary results. Section 3 is the proof of Theorem 1. In Section 4, the notion of a subcritical pair is introduced, and the behavior of subcritical pairs with respect to doubling is determined, leading to the proof of Theorem 2 in Section 5. Section 6 contains the proof of Theorem 3. In Section 7 we revisit the notion of congruence normality and prove Theorem 4. In Section 8, Theorem 4 is applied to posets of regions of simplicial hyperplane arrangements to prove Theorems 25 and 26, and a short proof is given that Coxeter arrangements satisfy the hypotheses of Theorem 26. 


\section{Preliminaries}

In this section, definitions are given and preliminary results are proven or quoted.

An arrangement $\mathcal{A}$ is a finite nonempty collection of hyperplanes (codimension 1 linear subspaces) in $\mathbb{R}^{d}$. The complement of the union of the hyperplanes is disconnected, and the closures of its connected components are called regions. In this paper, all hyperplane arrangements are central, meaning that every hyperplane contains the origin. The rank of an arrangement is the dimension of the linear span of the normals to the hyperplanes. A central hyperplane arrangement is called simplicial if every region is a simplicial cone. The lattice of intersections $L(\mathcal{A})$ of a central arrangement $\mathcal{A}$ consists of all arbitrary intersections of hyperplanes, partially ordered by reverse inclusion. A unique minimal element is adjoined to make $L(\mathcal{A})$ a lattice. A lattice is called supersolvable if it posesses a maximal chain $C$ such that for any maximal chain $C^{\prime}$, the sublattice generated by $C \cup C^{\prime}$ is distributive. A central arrangement $\mathcal{A}$ is called supersolvable if $L(\mathcal{A})$ is a supersolvable lattice. The definition of canonical base region will be given Section 3. The supersolvable arrangements include Coxeter arrangements of types A and B, as well as graphic arrangements associated to chordal graphs. For more information on hyperplane arrangements, see $[2,9,19]$.

The fundamental object of study in this paper is the poset $\mathcal{P}(\mathcal{A}, B)$ of regions of $\mathcal{A}$ with respect to a fixed region $B$. Define $S\left(R_{1}, R_{2}\right)$ to be the set of hyperplanes separating $R_{1}$ from $R_{2}$. For any region $R$, the set $S(R):=S(R, B)$ is called the separating set of $R$. The poset of regions is a partial order on the regions with $R_{1} \leq R_{2}$ if and only if $S\left(R_{1}\right) \subseteq S\left(R_{2}\right)$. The fixed region $B$ is called the base region. Examples of posets of regions are given later, in the figures. For more details on this poset, see $[2,9]$. For now, note only the following: in $\mathcal{P}(\mathcal{A}, B)$, the region $B$ is the unique minimal element, and complementation of separating sets is an antiautomorphism which is denoted by $R \mapsto-R$. In particular, there is a unique maximal element $-B$.

The poset notation used here is standard. We mention only a few items: if $x<y$ in $P$ and there is no $z \in P$ with $x<z<y$, say $y$ covers $x$ and write $x<\cdot y$. The set of pairs $(x, y)$ such that $x<y$ is denoted $E(P)$. The "E" is to suggest "edge," as these pairs are the edges in the Hasse diagram for $P$. A subset $I \subseteq P$ is called an order ideal if $x \in I$ and $y \leq x$ imply $y \in I$, and an order filter if the dual condition holds.

A poset $E$ on the same ground set as $P$ is called an extension of $P$ if $a \leq_{P} b$ implies $a \leq_{E} b$. An extension is called linear if it is a total order. The order dimension $\operatorname{dim}(P)$ of a poset $P$ is the smallest $d$ so that $P$ can be written as an intersection, as relations, of $d$ linear extensions of $P$. Say $Q$ is a(n) (induced) subposet of $P$ if there is a one-to-one map $i: Q \rightarrow P$ such that $x \leq_{Q} y$ if and only 
if $i(x) \leq_{P} i(y)$. The order dimension of $P$ is also the smallest $d$ so that $P$ can be embedded as an induced subposet of $\mathbb{R}^{d}$. When $Q$ is an induced subposet of $P$, $\operatorname{dim}(Q) \leq \operatorname{dim}(P)$. In Section 4, we give a characterization of order dimension in terms of subcritical pairs, a slight variation of the usual critical pairs.

Given a poset $P$ and an order-convex subset $C \subseteq P$, the doubling $P[C]$ of $C$ in $P$ is a subset of the product $P \times \mathbf{2}$, as explained below. The poset $\mathbf{2}$ is a chain with two elements $1<2$. Let $X:=\{x \in P: x \geq c$ for some $c \in C\}$, and define

$$
P[C]:=[((P-X) \cup C) \times\{1\}] \cup(X \times\{2\}) .
$$

Loosely speaking, the set $C$ is "doubled" via a product with $\mathbf{2}$, and the other elements of $P$ are inserted into $P \times \mathbf{2}$ in the most natural way. This definition is due to Day [6] who showed that if $L$ is a lattice, then $L[C]$ is also a lattice. A congruence normal lattice is a lattice obtained from the one-element lattice by a finite sequence of doublings of convex sets.

A lattice $L$ is called join semi-distributive if for any $x, y, z \in L$

$$
x \vee y=x \vee z \text { implies } x \vee(y \wedge z)=x \vee y .
$$

A lattice is meet semi-distributive if the dual condition holds and semi-distributive if it is both join semi-distributive and meet semi-distributive.

For an arbitrary hyperplane arrangement $\mathcal{A}$ and base region $B$ there is the following "change of base" isomorphism.

Proposition 5. For any region $R$, the interval $[R,-B]_{\mathcal{P}(\mathcal{A}, B)}$ in $\mathcal{P}(\mathcal{A}, B)$ is isomorphic to the interval $[R,-B]_{\mathcal{P}(\mathcal{A}, R)}$ in $\mathcal{P}(\mathcal{A}, R)$.

The proof is elementary and appears as part of the proof of [2, Theorem 3.4]. Here it is sufficient to point out that the isomorphism from $[R,-B]_{\mathcal{P}(\mathcal{A}, B)}$ to $[R,-B]_{\mathcal{P}(\mathcal{A}, R)}$ is to remove the set $S(R)$ from the separating set of each region in $[R,-B]_{\mathcal{P}(\mathcal{A}, B)}$.

Another useful result from [2] characterizes the join operation when $\mathcal{P}(\mathcal{A}, B)$ is a lattice. There is a closure operator defined on subsets of $\mathcal{A}$ in Section 5 of [2]. The details are not necessary here, as we will only need the fact that the separating sets of regions are closed sets and the fact that intersections of closed sets are closed. The closure of a set $S$ is written $\bar{S}$. The following is Theorem 5.5(2) of [2]:

Theorem 6. If $\mathcal{P}(\mathcal{A}, B)$ is a lattice and $R_{1}$ and $R_{2}$ are regions, then

$$
S\left(R_{1} \vee R_{2}\right)=\overline{S\left(R_{1}\right) \cup S\left(R_{2}\right)}
$$


Let $P$ be a finite poset with an equivalence relation $\Theta$ defined on the elements of $P$. Given $a \in P$, let $[a]_{\Theta}$ denote the equivalence class of $a$. The equivalence relation is an order congruence if:

(i) Every equivalence class is an interval.

(ii) The projection $\pi_{\downarrow}: P \rightarrow P$, mapping each element $a$ of $P$ to the minimal element in $[a]_{\Theta}$, is order-preserving.

(iii) The projection $\pi^{\uparrow}: P \rightarrow P$, mapping each element $a$ of $P$ to the maximal element in $[a]_{\Theta}$, is order-preserving.

Define a partial order on the congruence classes by $[a]_{\Theta} \leq[b]_{\Theta}$ if and only if there exists $x \in[a]_{\Theta}$ and $y \in[b]_{\Theta}$ such that $x \leq_{P} y$. The set of equivalence classes under this partial order is $P / \Theta$, the quotient of $P$ with respect to $\Theta$. It is convenient to identify $P / \Theta$ with the induced subposet $Q:=\pi_{\downarrow}(P)$. Such a subposet $Q$ is called an order quotient of $P$. It is easily seen that $\pi^{\uparrow}$ maps $Q$ isomorphically onto $\pi^{\uparrow}(P)$. The inverse is $\pi_{\downarrow}$. For more information on order congruences and quotients, see $[5,21]$.

A lattice congruence is an equivalence relation on a lattice which respects joins and meets. Specifically, if $a_{1} \equiv a_{2}$ and $b_{1} \equiv b_{2}$ then $a_{1} \vee b_{1} \equiv a_{2} \vee b_{2}$ and similarly for meets. When $P$ is a finite lattice, order congruences are exactly lattice congruences, and quotients with respect to lattice congruences are called lattice quotients.

Several times in this paper, it will be necessary to consider the deletion of a single hyperplane from an arrangement. We now establish some notation which will be used throughout the paper. Let $\mathcal{A}$ be a central arrangement, $H$ a hyperplane, and $B$ a fixed region of $\mathcal{A}$. Let $\mathcal{A}^{-}$be $\mathcal{A}-\{H\}$ and for any region $R$ of $\mathcal{A}$, let $R^{-}$be the region of $\mathcal{A}^{-}$containing $R$. Define inclusions $i^{\uparrow}, i_{\downarrow}: \mathcal{P}\left(\mathcal{A}^{-}, B^{-}\right) \rightarrow \mathcal{P}(\mathcal{A}, B)$ as follows. Any region of $\mathcal{A}^{-}$contains one or two regions of $\mathcal{A}$, and if there are two regions, one covers the other in $\mathcal{P}(\mathcal{A}, B)$. If a region $R$ of $\mathcal{A}^{-}$contains a unique region of $\mathcal{A}$, let $i^{\uparrow}$ and $i_{\downarrow}$ both map $R$ to that region. If $R$ contains two regions of $\mathcal{A}$, let $i^{\uparrow}$ map $R$ to the "higher" region in $\mathcal{P}(\mathcal{A}, B)$, and $i_{\downarrow}$ map $R$ to the lower.

Also, define projections $\pi^{\uparrow}$ and $\pi_{\downarrow}$ mapping $\mathcal{P}(\mathcal{A}, B)$ into itself. Define $\pi^{\uparrow}$ to map a region $R$ of $\mathcal{A}$ to $i^{\uparrow}\left(R^{-}\right)$, and let $\pi_{\downarrow}$ map $R$ to $i_{\downarrow}\left(R^{-}\right)$. Proposition 7 below explains when these maps define an order quotient. When we can do so without causing confusion, we will write $i^{\uparrow} R$ instead of $i^{\uparrow}(R)$, to avoid an overabundance of parentheses, and similarly for $i_{\downarrow}, \pi^{\uparrow}$ and $\pi_{\downarrow}$.

The poset $\mathcal{P}(\mathcal{A}, B)$ is an induced subposet of $\mathcal{P}\left(\mathcal{A}^{-}, B^{-}\right) \times \mathbf{2}$. Specifically, let $C$ be the set of regions of $\mathcal{A}^{-}$whose interiors intersect $H$. Let $U$ (for "up") be the set of regions in $\mathcal{P}\left(\mathcal{A}^{-}, B^{-}\right)-C$ which are separated from $B$ by $H$ and let $D$ (for "down") be $\mathcal{P}\left(\mathcal{A}^{-}, B^{-}\right)-C-U$. Then

$$
\mathcal{P}(\mathcal{A}, B) \cong((D \cup C) \times\{1\}) \cup((U \cup C) \times\{2\}) .
$$


Proposition 7. The following are equivalent:

(i) $i_{\downarrow} \mathcal{P}\left(\mathcal{A}^{-}, B^{-}\right)$is an order quotient of $\mathcal{P}(\mathcal{A}, B)$, via the maps $\pi_{\downarrow}$ and $\pi^{\uparrow}$.

(ii) $C$ is order-convex in $\mathcal{P}\left(\mathcal{A}^{-}, B^{-}\right)$.

(iii) $\mathcal{P}(\mathcal{A}, B)$ is the doubling $\mathcal{P}\left(\mathcal{A}^{-}, B^{-}\right)[C]$ of $C$ in $\mathcal{P}\left(\mathcal{A}^{-}, B^{-}\right)$.

(iv) $i_{\downarrow}$ embeds $\mathcal{P}\left(\mathcal{A}^{-}, B^{-}\right)$as a subposet of $\mathcal{P}(\mathcal{A}, B)$.

(v) $i^{\uparrow}$ embeds $\mathcal{P}\left(\mathcal{A}^{-}, B^{-}\right)$as a subposet of $\mathcal{P}(\mathcal{A}, B)$.

Proof. Suppose that $C$ is not order-convex. Thus there are regions $R_{1}, R_{2}, R_{3}$ of $\mathcal{A}^{-}$so that $R_{1} \geq R_{2} \geq R_{3}$, with $R_{1}, R_{3} \in C$ but $R_{2} \notin C$. Thus $i^{\uparrow} R_{1}>i_{\downarrow} R_{1}$, $i^{\uparrow} R_{2}=i_{\downarrow} R_{2}$ and $i^{\uparrow} R_{3}>i_{\downarrow} R_{3}$. If $H \in S\left(i^{\uparrow} R_{2}\right)$, then $i^{\uparrow} R_{1} \geq i^{\uparrow} R_{2}$, but

$$
i_{\downarrow} R_{1}=\pi_{\downarrow} i^{\uparrow} R_{1} \geq \pi_{\downarrow} i^{\uparrow} R_{2}=i_{\downarrow} R_{2} .
$$

If $H \notin S\left(i^{\uparrow} R_{2}\right)$, then $i^{\uparrow} R_{2} \geq i_{\downarrow} R_{3}$ but

$$
i^{\uparrow} R_{2}=\pi^{\uparrow} i^{\uparrow} R_{2} \geq \pi^{\uparrow} i_{\downarrow} R_{3}=i^{\uparrow} R_{3} .
$$

In either case there is a contradiction of the definition of order quotient. Thus (i) implies (ii).

When $C$ is order-convex, it is easily seen that $\mathcal{P}(\mathcal{A}, B)$ and $\mathcal{P}\left(\mathcal{A}^{-}, B^{-}\right)[C]$ are exactly the same subposet of $\mathcal{P}\left(\mathcal{A}^{-}, B^{-}\right) \times \mathbf{2}$. Edelman [9] showed that in any maximal chain in $\mathcal{P}(\mathcal{A}, B)$, each cover relation corresponds to crossing one hyperplane, and, of course, every hyperplane is crossed. Therefore, the $X$ in the definition of doubling is exactly $U \cup C$ and $P-X$ is exactly $D$. Thus (ii) implies (iii).

The fact that doublings of lattices give rise to lattice quotients is well known. The proof for posets is similar. Suppose that $\mathcal{P}(\mathcal{A}, B)$ is $\mathcal{P}\left(\mathcal{A}^{-}, B^{-}\right)[C]$. Then $\pi_{\downarrow}$ can be thought of as a map to $\mathcal{P}\left(\mathcal{A}^{-}, B^{-}\right)$by ignoring the second entry in $\mathcal{P}(\mathcal{A}, B) \subseteq \mathcal{P}\left(\mathcal{A}^{-}, B^{-}\right) \times \mathbf{2}$, and is therefore easily seen to be order-preserving. By self-duality, $\pi^{\uparrow}$ is also order-preserving. Thus (iii) implies (i).

The fact that (iii) implies (iv) is elementary, and we omit the details. The equivalence of (iv) and (v) is clear by self-duality. Finally, we show that (iv) and (v) imply (ii). If (ii) fails then, as in the first paragraph of this proof, either Expression (1) or Expression (2) holds, violating either (iv) or (v).

Proposition 7 and the definition of congruence normality inspire the following recursive definition: Let $\mathcal{A}$ be an arrangement with base region $B$ and order the hyperplanes $H_{1}, H_{2}, \ldots, H_{k}$. Let $\mathcal{A}^{-}, B^{-}, C, i^{\uparrow}, i_{\downarrow}, \pi^{\uparrow}$ and $\pi_{\downarrow}$ be as defined above with respect to deleting the hyperplane $H=H_{k}$. Then $H_{1}, H_{2}, \ldots, H_{k}$ is a quotient ordering of $\mathcal{A}$ with respect to $B$ if $C$ is order-convex in $\mathcal{P}\left(\mathcal{A}^{-}, B^{-}\right)$and $H_{1}, H_{2}, \ldots, H_{k-1}$ is a quotient ordering of $\mathcal{A}^{-}$with respect to $B^{-}$. If $k=1$ then $H_{1}$ is a quotient ordering of $\mathcal{A}$ with respect to either choice of base region.

Not every hyperplane arrangement with a fixed base region has a quotient ordering, as shown by the example in Figure 1. The reader can verify that for the 
arrangement and base region in Figure 1, for any choice of $H$, the set of regions of $\mathcal{A}^{-}$whose interiors intersect $H$ is not order-convex in $\mathcal{P}\left(\mathcal{A}^{-}, B^{-}\right)$.

Remark 8. Explanation of the figures. Each figure represents a central hyperplane arrangement in $\mathbb{R}^{3}$ as an arrangement of great circles on a 2 -sphere. The left drawing shows the "northern hemisphere" of the sphere, and the right drawing is a 180-degree rotation of what would be seen if the northern hemisphere were removed. The advantage of the 180-degree rotation is that the two drawings are identical except for the labeling of the regions, and the antipodal map corresponds to translating one drawing on top of the other. If a solid line is shown at the equator, then the equatorial plane is part of the arrangement. Otherwise, the equator is shown as a dotted line. The base region $B$ is marked, and the other regions are labeled by their separating sets. To reduce clutter, the hyperplanes themselves are not numbered, but the numbering of the hyperplanes will be apparent from the labeling of the regions.
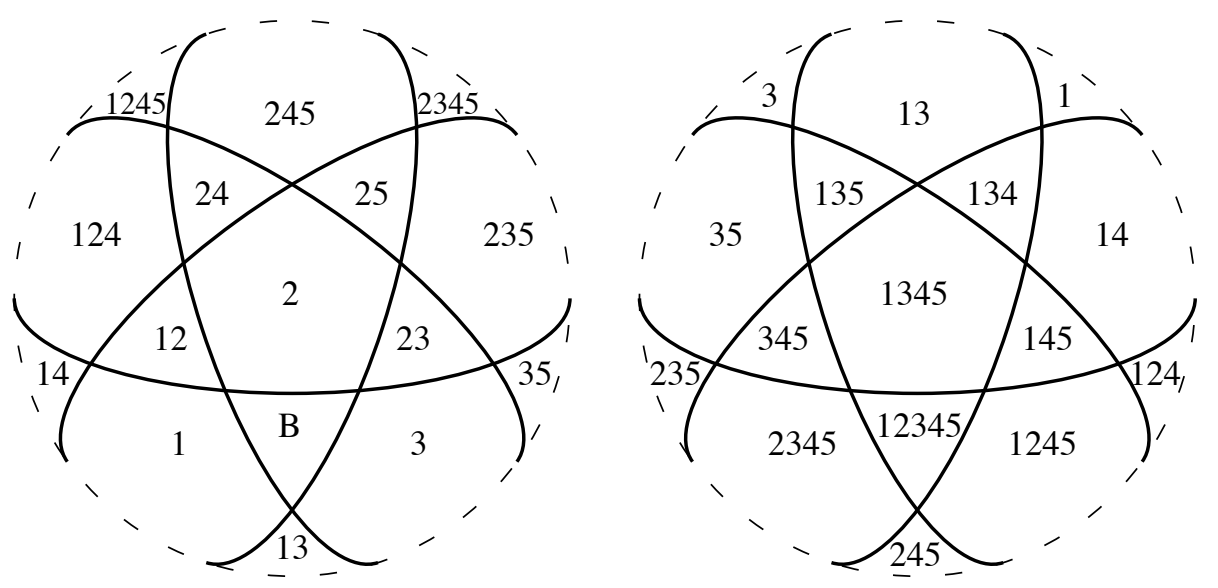

Figure 1. A hyperplane arrangement $\mathcal{A}$ which has no quotient ordering with respect to $B$.

Proposition 7 immediately implies the following:

Proposition 9. If $\mathcal{A}$ admits a quotient ordering with respect to a base region $B$, then $\mathcal{P}(\mathcal{A}, B)$ is a congruence normal lattice.

There is a partial converse to Proposition 9. Define an arrangement $\mathcal{A}$ to be 3 -generic if no three hyperplanes of $\mathcal{A}$ intersect in a codimension 2 subspace. This 
definition is a special case of the notion of $k$-generic arrangements in [24]. The following proposition can be proven using Theorem 4 and a similar argument to that given in the proof of Theorem 25. We omit the details.

Proposition 10. If $\mathcal{A}$ is a 3-generic hyperplane arrangement and $B$ is a base region, then $\mathcal{P}(\mathcal{A}, B)$ is a congruence normal lattice if and only if $\mathcal{A}$ admits a quotient ordering with respect to $B$.

We would guess that the converse of Proposition 9 is false for general arrangements, but we are not aware of a counterexample. Given the difference in the hypotheses of Theorems 25 and 26 , such a counterexample probably exists among simplicial arrangements. In light of Proposition 10, the example in Figure 1 is not congruence normal.

This section concludes with an explanation of the right weak order on permutations. Let $S_{n}$ be the symmetric group on the set $[n]:=\{1,2, \ldots, n\}$, and write an element $\pi \in S_{n}$ as $\pi_{1} \pi_{2} \cdots \pi_{n}$, meaning that $i \mapsto \pi_{i}$. The inversion set $I(\pi)$ of $\pi$ is

$$
I(\pi):=\left\{\left(\pi_{i}, \pi_{j}\right): \pi_{i}<\pi_{j}, i>j\right\} .
$$

One definition of the right weak order is that $\pi \leq \tau$ if and only if $I(\pi) \subseteq I(\tau)$. This partial order is the poset of regions of a Coxeter arrangement of type A, with the inversion set $I$ corresponding to the separating set $S$. For more details, see [15].

\section{Supersolvable arrangements}

In this section, we quote a characterization of supersolvable hyperplane arrangements which appeared in [2], prove a few simple propositions and then prove Theorem 1. In the following theorem of Björner, Edelman and Ziegler [2], " $\uplus$ " refers to disjoint union. The uniqueness in part (ii) is not stated in [2], but follows immediately from the proof given there.

Theorem 11. [2, Theorem 4.3] Every hyperplane arrangement of rank 2 is supersolvable. A hyperplane arrangement $\mathcal{A}$ of rank $d \geq 2$ is supersolvable if and only if it can be written as $\mathcal{A}=\mathcal{A}_{0} \uplus \mathcal{A}_{1}$, where

(i) $\mathcal{A}_{0}$ is a supersolvable arrangement of rank $d-1$.

(ii) For any $H^{\prime}, H^{\prime \prime} \in \mathcal{A}_{1}$, there is a unique $H \in \mathcal{A}_{0}$ such that $H^{\prime} \cap H^{\prime \prime} \subseteq H$.

Given a fixed region $R$ of $\mathcal{A}_{0}$, consider the regions of $\mathcal{A}$ contained in $R$. As an immediate consequence of Theorem 3, the graph of adjacency on these regions is a path. Following [2], define a canonical base region inductively: Any region of an arrangement of rank 2 is a canonical base region. For a supersolvable arrangement $\mathcal{A}=\mathcal{A}_{0} \uplus \mathcal{A}_{1}$, and a region $R$ of $\mathcal{A}$, let $R_{0}$ be the region of $\mathcal{A}_{0}$ containing $R$. Then $B$ is a canonical base region if $B_{0}$ is a canonical base region of $\mathcal{A}_{0}$ and if the regions of 
$\mathcal{A}$ contained in $B_{0}$ are linearly ordered in $\mathcal{P}(\mathcal{A}, B)$. Figure 2 shows a supersolvable arrangement of rank 3 with canonical base region $B$.
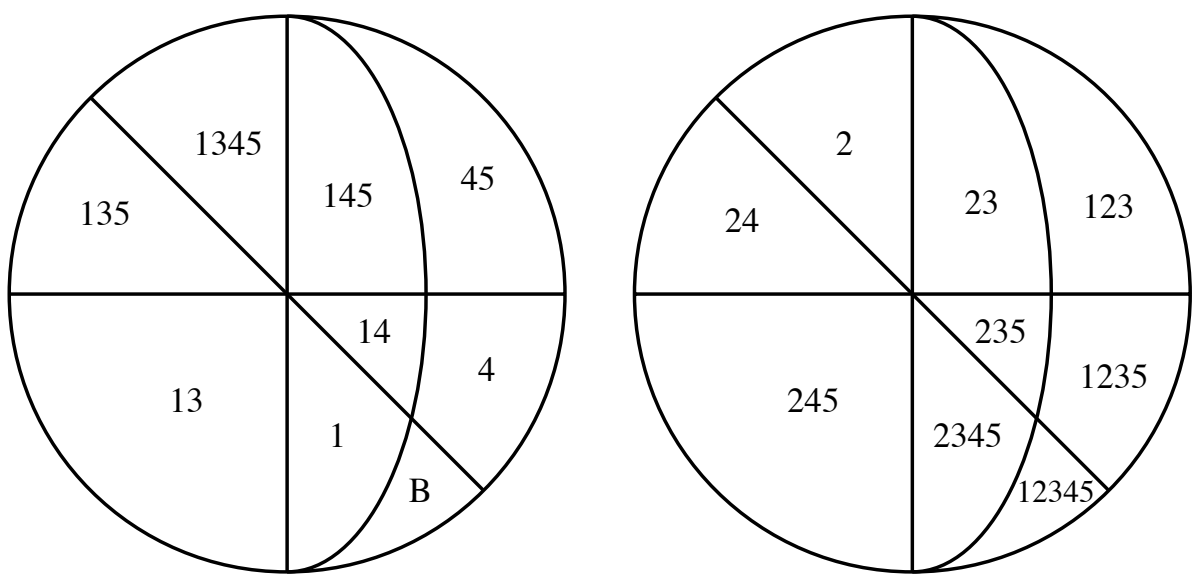

Figure 2. A supersolvable arrangement $\mathcal{A}$ with canonical base region $B$. Theorem 11 applies with $\mathcal{A}_{0}=\left\{H_{1}, H_{2}, H_{3}\right\}$.

Theorems 1 and 2 can be phrased in slightly more general terms. Call $\mathcal{A}$ a modular extension of $\mathcal{A}_{0}$ if $\mathcal{A}=\mathcal{A}_{0} \uplus \mathcal{A}_{1}$, such that condition (ii) of Theorem 11 is satisfied, and such that the rank of $\mathcal{A}_{0}$ is one less than the rank of $\mathcal{A}$. In more common hyperplane-arrangement terminology, this is the same as saying that $\mathcal{A}_{0}$ is the localization of $\mathcal{A}$ to a modular coatom of $L(\mathcal{A})$. If $B_{0}$ is the base region of $\mathcal{A}_{0}$, say $B$ is compatible with $B_{0}$ if the regions of $\mathcal{A}$ contained in $B_{0}$ are linearly ordered in $\mathcal{P}(\mathcal{A}, B)$. The following propositions all hold for the supersolvable case, and more generally for modular extensions.

The linear ordering of $\mathcal{A}$-regions contained in $B_{0}$ gives a corresponding linear ordering of the hyperplanes of $\mathcal{A}_{1}$. Using this linear ordering, write $\mathcal{A}_{1}$ as $H_{1}, H_{2}, \ldots H_{n}$, with $H_{1}$ bounding $B$. Given any region $R$, there is a linear ordering on the hyperplanes of $\mathcal{A}_{1}$, obtained in the same manner. Define a map $\eta$ from the regions of $\mathcal{A}_{0}$ to the symmetric group $S_{n}$, by sending each region $R$ of $\mathcal{A}_{0}$ to the permutation of $1,2, \ldots, n$ corresponding to the linear order on $H_{1}, H_{2}, \ldots H_{n}$ in $R$.

Proposition 12. The map $\eta$ is order-preserving from $\mathcal{P}\left(\mathcal{A}_{0}, B_{0}\right)$ to the right weak order on $S_{n}$.

Proof. Suppose that $R_{1} \in \mathcal{P}\left(\mathcal{A}_{0}, B_{0}\right)$, and suppose that the elements $i$ and $j$ form an inversion in $\eta\left(R_{1}\right)$. By definition, $\eta\left(B_{0}\right)$ is the permutation $12 \cdots n$. Since $i$ and $j$ form an inversion in $\eta\left(R_{1}\right)$, but not in $\eta\left(B_{0}\right)$, there is some $H \in S_{0}\left(R_{1}\right)$ such that 
$H_{i} \cap H_{j} \subseteq H$. Here $S_{0}$ denotes the separating set with respect to $\mathcal{P}\left(\mathcal{A}_{0}, B_{0}\right)$. By the uniqueness in Theorem 11(ii), there is no other hyperplane in $\mathcal{A}_{0}$ containing $H_{i} \cap H_{j}$. In particular, for any $R_{2} \geq R_{1}, H \notin S_{0}\left(R_{1}, R_{2}\right)$, so $i$ and $j$ form an inversion in $\eta\left(R_{2}\right)$ as well. Thus for any $R_{1} \leq R_{2}$, one has $I\left(\eta\left(R_{1}\right)\right) \subseteq I\left(\eta\left(R_{2}\right)\right)$, or in other words $\eta\left(R_{1}\right) \leq \eta\left(R_{2}\right)$ in the right weak order.

For $R$ a region of $\mathcal{A}_{0}$, define $\rho(R)$ to be the set of entries of $\eta(R)$ which do not form an inversion with the entry $n$. Proposition 12 immediately implies the following fact, where $\mathbf{2}^{n-1}$ is the Boolean lattice of subsets of $\{1,2, \ldots, n-1\}$.

Proposition 13. The map $\rho: \mathcal{P}\left(\mathcal{A}_{0}, B_{0}\right) \rightarrow \mathbf{2}^{n-1}$ is order-reversing.

Now use the notation defined above for deleting the hyperplane $H=H_{n}$ from $\mathcal{A}$. It is convenient to think of $\mathcal{P}\left(\mathcal{A}^{-}, B^{-}\right)$as a subposet of $\mathcal{P}\left(\mathcal{A}_{0}, B_{0}\right) \times \mathbf{2}^{n-1}$. This is done by writing the separating set of a region $R$ of $\mathcal{A}^{-}$as $S(R)=S_{0}\left(R_{0}\right) \cup S_{1}(R)$, where $S_{1}(R)$ is defined to be $S(R) \cap \mathcal{A}_{1}$, and $S_{0}$ denotes the separating set with respect to $\mathcal{P}\left(\mathcal{A}_{0}, B_{0}\right)$. The following proposition is the heart of Theorems 1 and 2 .

Proposition 14. The poset $\mathcal{P}(\mathcal{A}, B)$ is obtained from $\mathcal{P}\left(\mathcal{A}^{-}, B^{-}\right)$by doubling the convex set $\left\{(R, \rho(R)): R \in \mathcal{P}\left(\mathcal{A}_{0}, B_{0}\right)\right\}$. Furthermore, if $T_{1}$ and $T_{2}$ are both in the doubled set and $T_{1} \leq T_{2}$, then $S_{1}\left(T_{1}\right)=S_{1}\left(T_{2}\right)$.

Proof. It is apparent that, in the language of Proposition 7, the set $C$ of regions of $\mathcal{A}^{-}$whose interiors intersect $H_{n}$ is $\left\{(R, \rho(R)): R \in \mathcal{P}\left(\mathcal{A}_{0}, B_{0}\right)\right\}$. To prove that $C$ is convex, let $T_{1}, T_{2} \in C$ and let $T_{1} \leq T_{3} \leq T_{2}$ in $\mathcal{P}\left(A^{-}, B^{-}\right)$. Then $T_{1}$ is $\left(R_{1}, \rho\left(R_{1}\right)\right)$ for some region $R_{1}$ of $\mathcal{A}_{0}$ and similarly, $T_{2}$ is $\left(R_{2}, \rho\left(R_{2}\right)\right)$. Since $T_{1} \leq T_{2}$, then also $R_{1} \leq R_{2}$ in $\mathcal{P}\left(\mathcal{A}_{0}, B_{0}\right)$ and $\rho\left(R_{1}\right) \subseteq \rho\left(R_{2}\right)$. But also, since $\rho$ is order-reversing and $R_{1} \leq R_{2}$, then $\rho\left(R_{1}\right) \supseteq \rho\left(R_{2}\right)$, and therefore $\rho\left(R_{1}\right)=\rho\left(R_{2}\right)$. This proves the assertion that $S_{1}\left(T_{1}\right)=S_{1}\left(T_{2}\right)$. But $S_{1}\left(T_{1}\right) \subseteq S_{1}\left(T_{3}\right) \subseteq S_{1}\left(T_{2}\right)$, so it is possible to write $T_{3}=\left(R_{3}, \rho\left(R_{1}\right)\right)$ for some region $R_{3}$ of $\mathcal{A}_{0}$, with $R_{1} \leq R_{3} \leq R_{2}$ in $\mathcal{P}\left(\mathcal{A}_{0}, B_{0}\right)$. Applying the map $\rho$ shows that $\rho\left(R_{3}\right)$ is contained in $\rho\left(R_{1}\right)$ and contains $\rho\left(R_{2}\right)$. But $\rho\left(R_{1}\right)=\rho\left(R_{2}\right)$, so $\rho\left(R_{3}\right)=\rho\left(R_{1}\right)$ and therefore $T_{3}=\left(R_{3}, \rho\left(R_{3}\right)\right)$, or in other words $T_{3} \in C$.

Proposition 14 provides the inductive step in a proof of the following theorem which was already stated in Section 1.

Theorem 1. The poset of regions (with respect to a canonical base region) of a supersolvable hyperplane arrangement is a congruence normal lattice.

Specifically, given a quotient ordering of $\mathcal{A}_{0}$, a quotient ordering of $\mathcal{A}$ is obtained by appending, in order, the hyperplanes $H_{1}, H_{2}, \ldots, H_{n}$ to the end of the quotient ordering of $\mathcal{A}_{0}$. 
Since the propositions in this section do not depend on the assumption that $\mathcal{A}_{0}$ is a supersolvable arrangement, they also prove that if $\mathcal{A}$ is a modular extension of $\mathcal{A}_{0}$ and $B$ is compatible with $B_{0}$, then $\mathcal{P}(\mathcal{A}, B)$ is obtained from $\mathcal{P}\left(\mathcal{A}_{0}, B_{0}\right)$ by a sequence of doublings. Because a lattice $L$ is a quotient of the doubling $L[C]$, the proof of Theorem 1 also implies that if $\mathcal{A}$ is a supersolvable arrangement with canonical base region $B$, then $\mathcal{P}\left(\mathcal{A}_{0}, B_{0}\right)$ is a lattice quotient of $\mathcal{P}(\mathcal{A}, B)$. The same holds if $\mathcal{A}$ is a modular extension of $\mathcal{A}_{0}$ and $B$ is compatible with $B_{0}$. In particular, the weak order on $A_{n}$ is a lattice quotient of the weak order on $A_{n+1}$ and similarly for type B. This is easily proven by other methods for any parabolic subgroup of a finite Coxeter group [22].

Although supersolvable arrangements give rise to congruence normal lattices, there are non-supersolvable arrangements which also give rise to congruence normal lattices. One example appears later as Figure 3 in Section 6. It is easily seen that $H_{1}, H_{2}, H_{3}, H_{4}$ is a quotient ordering of the example in Figure 3. Also, supersolvable lattices can fail to be semi-distributive (thus failing to be congruence uniform), as can be seen in Figure 2, above. In Figure 2, referring to regions by their separating sets, it is seen that $45 \wedge 14=45 \wedge 24=4$, but $45 \wedge(14 \vee 24)=45 \wedge 12345=45$.

\section{Subcritical pairs}

This section contains preliminary definitions and results needed for the proof of Theorem 2. We define subcritical pairs and show that they are well-behaved with respect to order quotients, and in particular deal with the case where the quotient arises from the removal of a hyperplane from an arrangement.

In a poset $P$, if neither $x \leq y$ nor $x \geq y$, call $x$ and $y$ incomparable and write $x \| y$. Given $x \in P$, define

$$
\begin{aligned}
D(x) & :=\{y \in P: y<x\} \\
U(x) & :=\{y \in P: y>x\} \\
D[x] & :=\{y \in P: y \leq x\} \\
U[x] & :=\{y \in P: y \geq x\} .
\end{aligned}
$$

A critical pair in $P$ is $(a, b)$ with the following properties:

(i) $a \| b$,

(ii) $D(a) \subseteq D(b)$, and

(iii) $U(b) \subseteq U(a)$.

An extension $E$ of a poset $P$ is said to reverse a critical pair $(a, b)$ if $b<a$ in $E$. The order dimension of a poset $P$ is equal to the smallest $d$ such that there exist linear extensions $L_{1}, \ldots, L_{d}$ such that for each critical pair $(a, b)$ of $P$ there is some $L_{i}$ which reverses $(a, b)$. See $[20,23]$ for details. 
The critical digraph $\mathcal{D}(P)$ of $P$ is the directed graph whose vertices are the critical pairs, with directed edge $(a, b) \rightarrow(c, d)$ whenever $b \geq c$. Directed edges in the critical digraph will be called critical arrows. The next proposition follows from Lemma 6.3 of Chapter 1 of [23].

Proposition 15. Let $S$ be any set of critical pairs of $P$. Then there is a linear extension of $P$ reversing every critical pair in $S$ if and only if the subgraph of $\mathcal{D}(P)$ induced by $S$ is acyclic.

The critical complex $\mathbf{C}(P)$ of $P$ is an abstract simplicial complex whose vertices are the critical pairs of $P$, and whose faces are the sets of vertices which induce acyclic subgraphs of $\mathcal{D}(P)$. A set of faces $\left\{F_{i}\right\}$ of a simplicial complex $\mathbf{C}$ with vertex set $V$ is a covering set if $\bigcup_{i} F_{i}=V$. Proposition 15 implies that (when $P$ is not a total order) the order dimension of $P$ is the size of a smallest covering set of $\mathbf{C}(P)$. Critical complexes also appear in [21].

As will be seen below, when order quotients are involved, it is more convenient to work with subcritical pairs. A pair $(a, b)$ is called subcritical if:

(i') $a \not z b$,

(ii') $D(a) \subseteq D[b]$, and

(iii') $U(b) \subseteq U[a]$.

Critical pairs are also subcritical, and a subcritical pair $(a, b)$ is critical if and only if $a \ngtr b$. If $(a, b)$ is a subcritical pair that is not critical, then $a$ covers $b$ and nothing else, and $b$ is covered by $a$ and by nothing else. The set of subcritical pairs of $P$ is denoted $\operatorname{Subcrit}(P)$. Readers familiar with the double-arrow relation " $\uparrow$ " on a lattice (see for example [3]) will notice that subcritical pairs are a generalization of the double-arrow relation to general posets.

If $(a, b)$ is a subcritical pair that is not critical, every extension of $P$ reverses $(a, b)$. Thus the order dimension of $P$ is also the smallest $d$ so that there exist linear extensions $L_{1}, \ldots, L_{d}$ such that any subcritical pair $(a, b)$ of $P$ is reversed in some $L_{i}$. There are analogous definitions of the subcritical digraph $\mathcal{D}_{s}(P)$ and the subcritical complex $\mathbf{C}_{s}(P)$, and the analog of Proposition 15 holds, so the order dimension of $P$ is the size of a smallest covering set of $\mathbf{C}_{s}(P)$.

Proposition 16. Suppose $Q$ is an order quotient of $P$, and $(a, b)$ is subcritical in $P$. Then $\left(\pi_{\downarrow} a, \pi_{\downarrow} b\right)$ satisfies conditions (ii') and (iii') in the definition of subcritical pair in $Q$. Also, $\left(\pi_{\downarrow} a, \pi_{\downarrow} b\right)$ is subcritical in $Q$ if and only if $a \in Q$, if and only if $b \in \pi^{\uparrow} Q$.

Proof. Suppose $(a, b)$ is subcritical in $P$. If $x \in Q$ has $x<\pi_{\downarrow} a$, then $x \leq b$ because $(a, b)$ is subcritical in $P$. Thus $x=\pi_{\downarrow} x \leq \pi_{\downarrow} b$. Suppose $x \in Q$ has $\pi_{\downarrow} b<x$, so that $b \leq \pi^{\uparrow}\left(\pi_{\downarrow} b\right) \leq \pi^{\uparrow} x$. Notice that $b<\pi^{\uparrow} x$, because if $b=\pi^{\uparrow} x$ then $\pi_{\downarrow} b=x$. Because $(a, b)$ is subcritical in $P, a \leq \pi^{\uparrow} x$, and so $\pi_{\downarrow} a \leq \pi_{\downarrow}\left(\pi^{\uparrow} x\right)=x$. Thus $\left(\pi_{\downarrow} a, \pi_{\downarrow} b\right)$ satisfies conditions $\left(\mathrm{ii}^{\prime}\right)$ and $\left(\mathrm{iii}^{\prime}\right)$ in the definition of a subcritical pair in $Q$. 
Suppose that $a \notin Q$ and therefore $\pi_{\downarrow} a<a$. Then $\pi_{\downarrow} a \leq b$ because $(a, b)$ is subcritical, and therefore $\pi_{\downarrow} a \leq \pi_{\downarrow} b$, so $\left(\pi_{\downarrow} a, \pi_{\downarrow} b\right)$ is not subcritical in $Q$. On the other hand, suppose $a \in Q$. Then $a \not \leq \pi_{\downarrow} b$, because $a \not \leq b$ and $\pi_{\downarrow} b \leq b$. If $b \neq \pi^{\uparrow} b$ then $b<\pi^{\uparrow} b$, and therefore $a \leq \pi^{\uparrow} b$. So also $a=\pi_{\downarrow} a \leq \pi_{\downarrow}\left(\pi^{\uparrow} b\right)$. But since $\pi_{\downarrow}\left(\pi^{\uparrow} b\right)=\pi_{\downarrow} b \leq b$, this implies that $a \leq b$, a contradiction of condition ( $\left.\mathrm{i}^{\prime}\right)$ which shows that $b=\pi^{\uparrow} b \in \pi^{\uparrow} Q$. If $b \in \pi^{\uparrow} Q$, the dual argument shows that $a \in Q$. Since $a \not \leq b$ and $\pi_{\downarrow} b \leq b, a \not \leq \pi_{\downarrow} b$. So $\left(a, \pi_{\downarrow} b\right)=\left(\pi_{\downarrow} a, \pi_{\downarrow} b\right)$ is subcritical in $Q$.

Proposition 17. Suppose $Q$ is an order quotient of $P$, and $(a, b)$ is critical (respectively subcritical) in $Q$. Then $\left(a, \pi^{\uparrow} b\right)$ is critical (respectively subcritical) in $P$.

Proof. Suppose $(a, b)$ is subcritical in $Q$. Then $a \not \leq b$ in $P$ because $Q$ is an induced subposet. If $x \in P$ has $x<a$ then $\pi_{\downarrow} x<a$, so $\pi_{\downarrow} x \leq b$ because $(a, b)$ is subcritical in $Q$. Then $x \leq \pi^{\uparrow} x=\pi^{\uparrow}\left(\pi_{\downarrow} x\right) \leq \pi^{\uparrow} b$, and thus $D_{P}(a) \subseteq D_{P}\left[\pi^{\uparrow} b\right]$. If $x \in P$ has $x>\pi^{\uparrow} b$ then $\pi_{\downarrow}(x)>\pi_{\downarrow}\left(\pi^{\uparrow} b\right)=b$, so $\pi_{\downarrow} x \geq a$ because $(a, b)$ is subcritical in $Q$. Thus $x \geq \pi_{\downarrow} x \geq a$, and $\left(a, \pi^{\uparrow} b\right)$ is subcritical in $P$.

Suppose that $(a, b)$ is critical in $Q$ or equivalently, that $(a, b)$ is subcritical and $a \ngtr b$. Then by the previous paragraph, $\left(a, \pi^{\uparrow} b\right)$ is subcritical in $P$. Since $\pi^{\uparrow} b \geq b$ and $a \nsupseteq b$, we have $a \nsupseteq \pi^{\uparrow} b$, and therefore $\left(a, \pi^{\uparrow} b\right)$ is critical in $P$.

The word "subcritical" cannot be replaced with "critical" in Proposition 16. For example, let $P$ be $\mathbf{2} \times \mathbf{2}$, let $Q=\{1\} \times \mathbf{2}$ and let $(a, b)=((1,2),(2,1))$. Then $(a, b)$ is critical in $P$ with $a \in Q$ and $\left(a, \pi_{\downarrow} b\right)$ is subcritical but not critical in $Q$.

Let $\max (P)$ be the set of maximal elements of a poset $P$ and let $\min (P)$ be the set of minimal elements. If $H$ is a hyperplane whose removal from $\mathcal{A}$ satisfies the equivalent conditions of Proposition 7 , define

$$
\begin{aligned}
S C_{\text {old }}(\mathcal{P}(\mathcal{A}, B)) & :=\left\{\left(i_{\downarrow} X, i^{\uparrow} Y\right):(X, Y) \in \operatorname{Subcrit}\left(\mathcal{P}\left(\mathcal{A}^{-}, B^{-}\right)\right\}\right. \\
S C_{\text {new }}(\mathcal{P}(\mathcal{A}, B)) & :=\left\{\left(i^{\uparrow} X, i_{\downarrow} Y\right): X \in \min (C), Y \in \max (C), X \leq Y\right\} .
\end{aligned}
$$

The notations $S C_{\text {old }}$ and $S C_{\text {new }}$ are meant to suggest that some of the subcritical pairs of $\mathcal{P}(\mathcal{A}, B)$ arise in a natural way from subcritical pairs of $\mathcal{P}\left(\mathcal{A}^{-}, B^{-}\right)$, while others are new.

Lemma 18. Let $H$ be a hyperplane whose removal from $\mathcal{A}$ satisfies the equivalent conditions of Proposition 7 for the base region $B$. Then

$$
\operatorname{Subcrit}(\mathcal{P}(\mathcal{A}, B))=S C_{\text {old }} \uplus S C_{\text {new }} .
$$

Proof. By Propositions 16 and 17, the set of subcritical pairs $(X, Y)$ in $\mathcal{P}(\mathcal{A}, B)$ with $X \in i_{\downarrow}\left(\mathcal{P}\left(\mathcal{A}^{-}, B^{-}\right)\right)=\pi_{\downarrow}(\mathcal{P}(\mathcal{A}, B))$ is exactly

$$
\left\{\left(X, \pi^{\uparrow} Y\right):(X, Y) \in \operatorname{Subcrit}\left(\pi_{\downarrow} \mathcal{P}(\mathcal{A}, B)\right)\right\} .
$$

This set is $\mathcal{S} C_{\text {old }}$. 
Let $X \in \min (C), Y \in \max (C)$ and $X \leq Y$ in $\mathcal{P}\left(\mathcal{A}^{-}, B^{-}\right)$. Then $i^{\uparrow} X \not \leq i_{\downarrow} Y$. Any $Z \in \mathcal{P}(\mathcal{A}, B)$ with $Z<i^{\uparrow} X$ has $H \notin S(Z)$ and thus $Z \leq i_{\downarrow} Y$. Otherwise, somewhere weakly below $Z^{-}$in $\mathcal{P}\left(\mathcal{A}^{-}, B^{-}\right)$there would be a region $R$ with $i^{\uparrow} R>i_{\downarrow} R$. But then $R \in C$, contradicting the minimality of $X$. Similarly, any $Z \in \mathcal{P}(\mathcal{A}, B)$ with $i_{\downarrow} Y<Z$ has $H \in Z$, so $i^{\uparrow} X<Z$. Thus $\left(i^{\uparrow} X, i_{\downarrow} Y\right) \in \operatorname{Subcrit}(\mathcal{P}(\mathcal{A}, B))$. It is now proven that $S C_{\text {new }} \subseteq \operatorname{Subcrit}(\mathcal{P}(\mathcal{A}, B))$.

Finally, it is sufficient to show that $\operatorname{Subcrit}(\mathcal{P}(\mathcal{A}, B))-S C_{\text {old }} \subseteq S C_{\text {new }}$. Suppose that $(X, Y) \in \operatorname{Subcrit}(\mathcal{P}(\mathcal{A}, B))$ and $X \notin i_{\downarrow}\left(\mathcal{P}\left(\mathcal{A}^{-}, B^{-}\right)\right)=\pi_{\downarrow} \mathcal{P}(\mathcal{A}, B)$. By Proposition 16, $X \notin \pi_{\downarrow} \mathcal{P}(\mathcal{A}, B)$ implies that $Y \notin \pi^{\uparrow} \mathcal{P}(\mathcal{A}, B)$. So $\pi_{\downarrow} X<X$ and $\pi^{\uparrow} Y>Y$ and therefore both $X^{-}$and $Y^{-}$are elements of $C$. Suppose $Z \in C$ has $Y^{-}<Z$ in $\mathcal{P}\left(\mathcal{A}^{-}, B^{-}\right)$. Then $Y=i_{\downarrow}\left(Y^{-}\right)<i_{\downarrow} Z$ in $\mathcal{P}(\mathcal{A}, B)$ and by subcriticality of $(X, Y)$, $X \leq i_{\downarrow} Z$. But this is impossible since $H \in S(X)$ but $H \notin S\left(i_{\downarrow} Z\right)$. Therefore, $Y^{-} \in \max (C)$. Since $\pi^{\uparrow} Y>Y$ we have $\pi^{\uparrow} Y \geq X$ and therefore $Y^{-} \leq X^{-}$, so $(X, Y)=\left(i^{\uparrow}\left(X^{-}\right), i_{\downarrow}\left(Y^{-}\right)\right) \in S C_{\text {new }}$.

It is easily checked that the subcritical arrows among $S C_{\text {old }}$ correspond exactly to the subcritical arrows among $\operatorname{Subcrit}\left(\mathcal{P}\left(\mathcal{A}^{-}, B^{-}\right)\right)$. Therefore:

Proposition 19. The subcomplex of $\mathbf{C}_{s}(\mathcal{P}(\mathcal{A}, B))$ induced by $S C_{\mathrm{old}}$ is isomorphic to $\mathbf{C}_{s}\left(\mathcal{P}\left(\mathcal{A}^{-}, B^{-}\right)\right)$.

\section{Order dimension of supersolvable arrangements}

In this section, after a preliminary proposition, we state and prove Theorem 2 . Let $\mathcal{A}=\mathcal{A}_{0} \uplus \mathcal{A}_{1}$ be a supersolvable arrangement with canonical base region $B$, and continue the notation from Section 3. Think of $\mathcal{P}(\mathcal{A}, B)$ as a subposet of $\mathcal{P}\left(\mathcal{A}_{0}, B_{0}\right) \times \mathbf{2}^{n}$, by writing the separating set of a region $R$ of $\mathcal{A}$ as $S(R)=$ $S_{0}\left(R_{0}\right) \cup S_{1}(R)$. Here, again, $S_{1}(R)$ is defined to be $S(R) \cap \mathcal{A}_{1}$ and $S_{0}$ denotes the separating set which defines $\mathcal{P}\left(\mathcal{A}_{0}, B_{0}\right)$.

Given a subset $S$ of $[n]$, write $S$ as a word of length $n$ in the alphabet $\{0,1\}$, so that for example $\{2,3,7\} \subset[7]$ is written as 0110001 . Define a total order on the subsets of $[n]$ by taking the lexicographic order on these words, reading from left to right, with $0<1$. Think of the sets $S_{1}(R)$ as subsets of $[n]$, by associating $H_{i}$ with $i$. Define a relation " $\preceq$ " on the regions of $\mathcal{A}$ such that $R_{1} \preceq R_{2}$ if and only if $S_{1}\left(R_{1}\right) \leq S_{1}\left(R_{2}\right)$ in this lexicographic order. Notice that " $\preceq$ " is reflexive and transitive, but not antisymmetric, and that any two regions of $\mathcal{A}$ are related

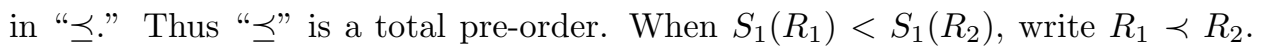
In other words $R_{1} \prec R_{2}$ when $R_{1} \preceq R_{2}$ but $R_{2} \npreceq R_{1}$. Note that if $R_{1} \leq R_{2}$ in $\mathcal{P}(\mathcal{A}, B)$, then $S_{1}\left(R_{1}\right) \subseteq S_{1}\left(R_{2}\right)$ and therefore $R_{1} \preceq R_{2}$.

By Theorem 1 and repeated applications of Lemma 18, the subcritical pairs of $\mathcal{P}\left(\mathcal{A}_{0}, B_{0}\right)$ can be thought of as a subset of $\operatorname{Subcrit}(\mathcal{P}(\mathcal{A}, B))$. Call this subset $S C_{0}$. 
Proposition 20. Let $\mathcal{A}$ be a modular extension of $\mathcal{A}_{0}$ and let $B$ be compatible with $B_{0}$. Let $(J, M) \in \operatorname{Subcrit}(\mathcal{P}(\mathcal{A}, B))-S C_{0}$. Then $M \prec J$.

Proof. The proof is by induction on $n$. Consider deleting the hyperplane $H=H_{n}$ and use the notation established previously for removal of a hyperplane, including the notation of Lemma 18. If $n=1$, since $\mathcal{A}$ has rank strictly greater than the rank of $\mathcal{A}_{0}$, then $\mathcal{P}(\mathcal{A}, B)$ is obtained by doubling the entire poset $\mathcal{P}\left(\mathcal{A}^{-}, B^{-}\right)=$ $\mathcal{P}\left(\mathcal{A}_{0}, B_{0}\right)$. Also $S C_{0}=S C_{\text {old }}$, so $(J, M)=\left(i^{\uparrow}\left(B^{-}\right), i_{\downarrow}\left(-B^{-}\right)\right) \in S C_{\text {new }}$. In particular, $S_{1}(J)=\{1\}$ and $S_{1}(M)=\emptyset$, so $M \prec J$.

Suppose $n>1$, and $(J, M) \in S C_{\text {old }}$. Then $(J, M)=\left(i_{\downarrow}\left(J^{-}\right), i^{\uparrow}\left(M^{-}\right)\right)$, and $\left(J^{-}, M^{-}\right) \in \operatorname{Subcrit}\left(\mathcal{P}\left(\mathcal{A}^{-}, B^{-}\right)\right)$. Since $(J, M)$ does not arise from a subcritical pair of $\mathcal{P}\left(\mathcal{A}_{0}, B_{0}\right)$, neither does $\left(J^{-}, M^{-}\right)$, so by induction $M^{-} \prec J^{-}$. Passing from $S_{1}\left(J^{-}\right)$to $S_{1}(J)$ involves only the new $n$th entry in the corresponding words, and similarly for $M^{-}$and $M$. Thus $M \prec J$ as well. Suppose on the other hand that $(J, M) \in S C_{\text {new }}$ and $n>1$. Then $(J, M)=\left(i^{\uparrow}\left(J^{-}\right), i_{\downarrow}\left(M^{-}\right)\right)$, and $J^{-}, M^{-} \in C$, with $J^{-} \leq M^{-}$. By Proposition 14, $S_{1}\left(J^{-}\right)=S_{1}\left(M^{-}\right)$. Thus the words for $S_{1}(J)$ and $S_{1}(M)$ are identical until the last entry, where $S_{1}(J)$ has a "1" and $S_{1}(M)$ has a "0." In particular, $M \prec J$.

Theorem 2. The order dimension of the poset of regions (with respect to a canonical base region) of a supersolvable hyperplane arrangement is equal to the rank of the arrangement.

Proof. First, notice that adding in the hyperplane $H_{1}$ to $\mathcal{A}_{0}$, doubles the entire poset $\mathcal{P}\left(\mathcal{A}_{0}, B_{0}\right)$. It is well known that when a finite poset $P$ has a unique minimal element and a unique maximal element, $\operatorname{dim}(P \times \mathbf{2})=\operatorname{dim}(P)+1$. By repeated applications of Proposition $7, \mathcal{P}\left(\mathcal{A}_{0}, B_{0}\right) \times \mathbf{2}$ is a subposet of $\mathcal{P}(\mathcal{A}, B)$, so $\operatorname{dim}(\mathcal{P}(\mathcal{A}, B)) \geq \operatorname{dim}\left(\mathcal{P}\left(\mathcal{A}_{0}, B_{0}\right)\right)+1$.

Let $d$ be the dimension of $\mathcal{P}\left(\mathcal{A}_{0}, B_{0}\right)$. Thus $\mathbf{C}_{s}\left(\mathcal{P}\left(\mathcal{A}_{0}, B_{0}\right)\right)$ can be covered by $d$ faces. By repeated applications of Proposition 19, the subcomplex of $\mathbf{C}_{s}(\mathcal{P}(\mathcal{A}, B))$ induced by $S C_{0}$ is isomorphic to $\mathbf{C}_{s}\left(\mathcal{P}\left(\mathcal{A}_{0}, B_{0}\right)\right)$, and therefore can be covered by $d$ faces. It will now be shown that the remaining vertices in fact form a face of $\mathbf{C}_{s}(\mathcal{P}(\mathcal{A}, B))$. Suppose $\left(J_{1}, M_{1}\right) \rightarrow\left(J_{2}, M_{2}\right)$ is a subcritical arrow with $\left(J_{1}, M_{1}\right),\left(J_{2}, M_{2}\right) \in \operatorname{Subcrit}(\mathcal{P}(\mathcal{A}, B))-S C_{0}$. In other words, $J_{2} \leq M_{1}$, and therefore $J_{2} \preceq M_{1}$. By Proposition 20, $M_{1} \prec J_{1}$, and therefore $J_{2} \prec J_{1}$. Thus every subcritical arrow in $\operatorname{Subcrit}(\mathcal{P}(\mathcal{A}, B))-S C_{0}$ is dual to a strict order relation in the pre-order " $\preceq$," and therefore $\operatorname{Subcrit}(\mathcal{P}(\mathcal{A}, B))-S C_{0}$ induces an acyclic subgraph of the subcritical digraph of $\mathcal{P}(\mathcal{A}, B)$. This face, together with the $d$ faces which cover $S C_{0}$ give a covering of $\mathbf{C}_{s}(\mathcal{P}(\mathcal{A}, B))$ by $d+1$ faces. By induction, $d$ is the rank of $\mathcal{A}_{0}$, and therefore the dimension of $\mathcal{P}(\mathcal{A}, B)$ is the rank of $\mathcal{A}$. 


\section{Simplicial arrangements and semi-distributivity}

In this section, we prove Theorem 3, give some examples which give context to the theorem and discuss the possible significance of semi-distributivity for order dimension.

Theorem 3. The poset of regions (with respect to any base region) of a simplicial hyperplane arrangement is a semi-distributive lattice.

Proof. Let $\mathcal{A}$ be a simplicial hyperplane arrangement and let $B$ be any base region. The fact that $\mathcal{P}(\mathcal{A}, B)$ is a lattice is $[2$, Theorem 3.4]. We will prove that $\mathcal{P}(\mathcal{A}, B)$ is meet semi-distributive. Join semi-distributivity follows by the self-duality of $\mathcal{P}(\mathcal{A}, B)$.

Suppose that $X, Y$ and $Z$ are regions of $\mathcal{A}$, with $X \wedge Y=X \wedge Z$. By the change of base isomorphism (Proposition 5), we may as well assume that $X \wedge Y=$ $B$. Thus it is necessary to show that $X \wedge(Y \vee Z)=B$. Let $\mathcal{B}$ be the set of hyperplanes defining the facets of $B$, and for any region $R$, let $\alpha(R):=S(R) \cap \mathcal{B}$. For each $H \in \mathcal{B}$, the singleton $\{H\}$ defines a region, and therefore the statement $X \wedge R=B$ is equivalent to the statement $\alpha(X) \cap \alpha(R)=\emptyset$. Thus by hypothesis, $\alpha(X) \cap \alpha(Y)=\alpha(X) \cap \alpha(Z)=\emptyset$ and it is sufficient to show that $\alpha(X) \cap \alpha(Y \vee Z)=\emptyset$.

By Theorem $6, S(Y \vee Z)=\overline{S(Y) \cup S(Z)}$. For any $H \in \mathcal{B}$, the set $\mathcal{A}-\{H\}$ is the separating set of some region $T_{H}$, and thus is a closed set. The intersection $S$ of all such $S\left(T_{H}\right)$ for $H \in \mathcal{B}-(\alpha(Y) \cup \alpha(Z))$ is a closed set (although not necessarily the separating set of any region). But $S$ contains $S(Y) \cup S(Z)$ and so $S$ contains $\overline{S(Y) \cup S(Z)}$. Then

$$
\alpha(X) \cap \alpha(Y \vee Z) \subseteq \alpha(X) \cap(S \cap \mathcal{B})=\alpha(X) \cap(\alpha(Y) \cup \alpha(Z))=\emptyset .
$$

The two figures below put Theorem 3 in context. Figure 3 is an example of an arrangement whose poset of regions is a lattice, but is not semi-distributive. Figure 4 shows a non-simplicial arrangement whose poset of regions is a semidistributive lattice.

The semi-distributive property does have some connection to order dimension. More precisely, the semi-distributive property is closely related to subcritical pairs. Define an element $x$ in a poset $P$ to be join-irreducible if it is not the join of the set $D(x)$ of elements strictly below it. This definition makes sense even for a nonlattice: the join of $D(x)$ may not exist, in which case $x$ is join-irreducible. It is an easy proposition that for any subcritical pair $(a, b)$ in a poset $P, a$ is join-irreducible and $b$ is meet-irreducible. It is also easy to see that for every join-irreducible $a$, there is at least one $b$ so that $(a, b)$ is subcritical. There may not be a $b$ so that $(a, b)$ is critical. Although it is phrased differently, [12, Theorem 2.56] states that the meet semi-distributive property of a lattice is equivalent to the property that 
for any join-irreducible $a$ there is a unique $b$ so that $(a, b)$ is subcritical. The dual result also holds.
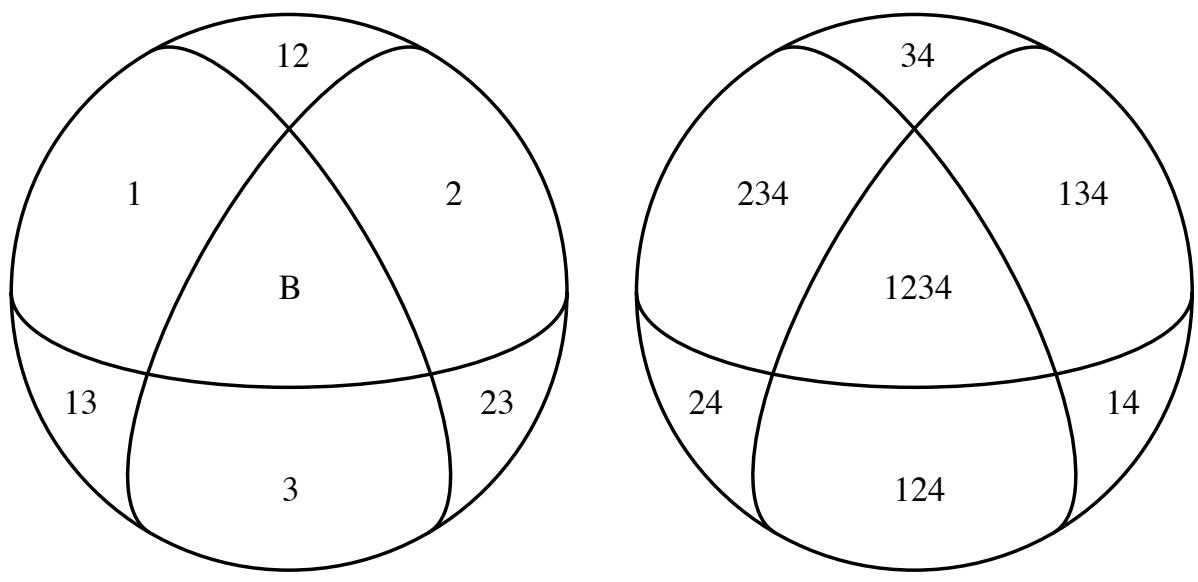

Figure 3. An arrangement $\mathcal{A}$ and base region $B$ such that $\mathcal{P}(\mathcal{A}, B)$ is a non-semi-distributive lattice.
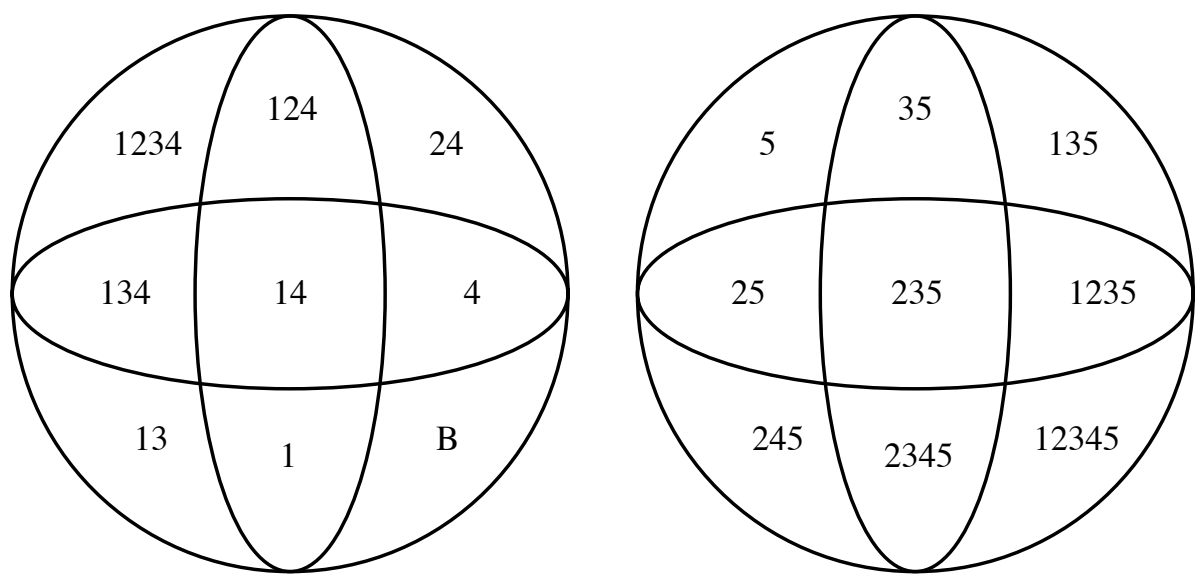

Figure 4. A non-simplicial arrangement $\mathcal{A}$ with base region $B$ such that $\mathcal{P}(\mathcal{A}, B)$ is a semi-distributive lattice. 


\section{Congruence normality}

Caspard, Le Conte de Poly-Barbut and Morvan [4] showed that the Cayley lattice (weak order) on a finite Coxeter group is congruence uniform. To do so, they defined an edge-labeling condition on a semi-distributive lattice which, when satisfied, implies a stronger property than congruence uniformity. This clever approach to congruence uniformity leads to the following question: is there an edge-labeling condition on a lattice which implies congruence normality, independent of semidistributivity? (Recall that a lattice is congruence uniform if and only if it is semi-distributive and congruence normal.) In this section we answer the question by defining CN-labelings, which will be used in Section 8 to discuss the congruence normality of posets of regions of simplicial arrangements.

We begin this section with Day's [7] definition of congruence normality, along with a slightly modified statement of his definition. Then we prove some preliminary propositions and prove Theorem 4. The proof of one direction of Theorem 4-namely that the existence of a CN-labeling implies congruence normality - is inspired by the proof given in [4].

The set of congruences Con $(L)$ of a lattice $L$, partially ordered by containment of relations, is known to be a distributive lattice. For any edge $x<y$ in a lattice $L$, define $\operatorname{Cg}(x, y)$ to be the smallest congruence relation on $L$ such that $x \equiv y$. For any edge $e, \operatorname{Cg}(e)$ is an element of $\operatorname{Irr}(\operatorname{Con}(L))$, the poset of join-irreducibles of the congruence lattice of $L$. This follows, for example from a characterization of the join in $\operatorname{Con}(L)$ which can be found in [13].

A join-irreducible $j$ in a lattice $L$ covers a unique element $j_{*}$, and a meetirreducible is covered by a unique element $m^{*}$. Day defined a lattice to be congruence normal if $\operatorname{Cg}\left(j_{*}, j\right)=\operatorname{Cg}\left(m, m^{*}\right)$ implies $j \not \leq m$, for any join-irreducible $j$ and meet-irreducible $m$. Day then proved that this property is equivalent to the property that the lattice can be obtained from the one-element lattice by a finite sequence of doublings of convex sets [7]. We will need the following equivalent definition of congruence normality. The "if" assertion is immediate by Day's definition, and the "only if" assertion is easily checked by induction on the number of doublings, and we omit the details.

Proposition 21. A lattice $L$ is congruence normal if and only if for any edges $\left(x_{1}, y_{1}\right)$ and $\left(x_{2}, y_{2}\right)$,

$$
\operatorname{Cg}\left(x_{1}, y_{1}\right)=\operatorname{Cg}\left(x_{2}, y_{2}\right) \text { implies } y_{1} \not \leq x_{2} .
$$

An interval $[x, y]$ is (join) fundamental if $y=a_{1} \vee a_{2}$ for some $a_{1}>x$ and $a_{2}>x$, with $a_{1} \neq a_{2}$. In a fundamental interval $[x, y]$, a pair of maximal chains $C_{1}$ and $C_{2}$ from $x$ to $y$ is called a fundamental pair if $a_{1} \in C_{1}$ and $a_{2} \in C_{2}$. Because $y=a_{1} \vee a_{2}$, the chains $C_{1}$ and $C_{2}$ are disjoint, except for the elements $x$ and $y$. Denote the 
element just below $y$ in $C_{1}$ by $b_{1}$ and similarly denote the element below $y$ in $C_{2}$ as $b_{2}$. Whenever a fundamental pair $C_{1}$ and $C_{2}$ are specified, these definitions of $x, y, a_{1}, a_{2}, b_{1}$ and $b_{2}$ will be assumed.

A $C N$-labeling of a finite lattice $L$ is a map $\gamma: E(L) \rightarrow P$, where $E(L)$ is the set of edges of $L$ and $P$ is some poset, such that for any fundamental pair $\left(C_{1}, C_{2}\right)$, the following requirements are satisfied:

(i) $\gamma\left(x, a_{1}\right)=\gamma\left(b_{2}, y\right)$

(ii) For any edge $e$ in $E\left(C_{1}\right)-\left\{\left(x, a_{1}\right),\left(b_{1}, y\right)\right\}, \gamma(e)<\gamma\left(x, a_{1}\right)$ and $\gamma(e)<\gamma\left(b_{1}, y\right)$.

(iii) The labels on $C_{1}$ are all distinct.

Since $\left(C_{2}, C_{1}\right)$ is also a fundamental pair, conditions (i) to (iii) also hold with the subscripts exchanged. One can require only weak inequalities in (ii), and the strictness is implied by condition (iii).

Given a lattice $L$ with a CN-labeling $\gamma: E(L) \rightarrow P$ and a lattice congruence $\Theta$, we can define a new labeling on the quotient lattice $L / \Theta$. If $E_{1}$ and $E_{2}$ are two congruence classes with $E_{1}<E_{2}$, then there are elements $x \in E_{1}$ and $a \in E_{2}$ with $x<a$. Define $\gamma_{\Theta}\left(E_{1}, E_{2}\right):=\gamma(x, a)$.

Proposition 22. The map $\gamma_{\Theta}: E(L / \Theta) \rightarrow P$ is well-defined and is a CN-labeling of $L / \Theta$.

Proof. Let $x \in E_{1}$ and $a_{2} \in E_{2}$ with $x<a_{2}$ in $L$. If $x$ is not the maximal element of $E_{1}$, then let $a_{1} \in E_{1}$ cover $x$, and let $y:=a_{1} \vee a_{2}$ in $L$. The interval $[x, y]_{L}$ is a fundamental interval, so let $C_{1}$ and $C_{2}$ be a fundamental pair in the interval, with $a_{1} \in C_{1}$ and $a_{2} \in C_{2}$, and let $b_{1}$ and $b_{2}$ be as usual. Since $E_{1}<\cdot E_{2}$, we have $y \in E_{2}$, so in particular $a_{2} \equiv y$ in $\Theta$. Therefore also $b_{1} \wedge a_{2} \equiv b_{1} \wedge y$ in $\Theta$, or in other words $x \equiv b_{1}$. Since $\gamma$ is a CN-labeling, $\gamma\left(x, a_{2}\right)=\gamma\left(b_{1}, y\right)$, and $b_{1}>x$. Proceeding inductively, we find that $\gamma\left(x, a_{2}\right)=\gamma(m, n)$ where $m$ is the maximal element of $E_{1}$ and $n \in E_{2}$ with $n>m$. Thus we can finish the proof that $\gamma_{\Theta}$ is well-defined by showing that there is a unique element of $E_{2}$ covering $m$. If there were two, say $n_{1}$ and $n_{2}$, then $n_{1} \wedge n_{2}$ is an element of $E_{2}$ which is above $m$, and therefore $n_{1} \wedge n_{2}=n_{1}=n_{2}$.

To show that $\gamma_{\Theta}$ is a CN-labeling, consider any fundamental interval $[X, Y]$ in $L / \Theta$, where $X$ and $Y$ are congruence classes, and $A_{1}$ and $A_{2}$ are congruence classes both covering $X$, with $Y=A_{1} \vee A_{2}$. Choose fundamental chains $C_{1}^{\prime}$ and $C_{2}^{\prime}$ in $[X, Y]$ and let $B_{1}$ be the congruence class in $C_{1}^{\prime}$ covered by $Y$ and similarly $B_{2}$. Let $x$ be the maximal element of $X$ and choose $a_{1} \in A_{1}$ and $a_{2} \in A_{2}$ both covering $x$. Let $y:=a_{1} \vee a_{2}$ and choose maximal chains $C_{1}$ and $C_{2}$ in $[x, y]$, containing respectively $a_{1}$ and $a_{2}$ such that each element of $C_{1}$ is in some equivalence class in $C_{1}^{\prime}$ and similarly for $C_{2}$. Then $C_{1}$ and $C_{2}$ are fundamental chains in the fundamental interval $[x, y]$. Let $b_{1}$ and $b_{2}$ be as usual. Then $b_{1} \in B_{1}$ because if $b_{1} \in Y$ then $b_{1} \equiv y$ in $\Theta$, which would imply that $a_{2} \wedge b_{1} \equiv a_{2} \wedge y$, or in other words $x \equiv a_{2}$, 
contradicting the fact that $x$ and $a_{2}$ are in different congruence classes. Similarly, $b_{2} \in B_{2}$. Now because $\gamma$ is a CN-labeling,

$$
\gamma_{\Theta}\left(X, A_{1}\right)=\gamma\left(x, a_{1}\right)=\gamma\left(b_{2}, y\right)=\gamma_{\Theta}\left(B_{2}, Y\right),
$$

and conditions (ii) and (iii) also hold for $\gamma_{\Theta}$ because the set of labels on $C_{1}^{\prime}$ is a subset of the set of labels on $C_{1}$.

An edge contraction on a lattice $L$ is an equivalence relation $\Theta$ such that each equivalence class has cardinality one or two. In other words, each equivalence class is an edge or a single element. Let $C$ be the set of two-element equivalence classes of $\Theta$.

Proposition 23. Let $\Theta$ be an edge contraction on $L$ with following properties:

(a) If $b \equiv t$ for some $b<t$ and $b^{\prime}>b$ for some $b^{\prime} \neq t$, then there is some $t^{\prime}$ with $b^{\prime} \equiv t^{\prime}$ and $t^{\prime}>t$.

(b) If $b \equiv t$ for some $b<t$ and $t^{\prime}<t$ for some $t^{\prime} \neq b$, then there is some $b^{\prime}$ with $b^{\prime} \equiv t^{\prime}$ and $b^{\prime}<b$.

Then $\Theta$ is a congruence, and furthermore $L \cong(L / \Theta)[C]$.

Proof. Suppose $b \equiv t$ in $\Theta$ for some $b<t$, and let $x \in L$. We need to show that $b \vee x \equiv t \vee x$, and dually. If $(b \vee x) \geq t$ then $b \vee x=t \vee x$, and if $x \leq b$ then $b \vee x=b \equiv t=t \vee x$. Otherwise choose some maximal chain of elements from $b$ to $b \vee x$. If $b \vee x>b$, then the element $t^{\prime}$ required in condition (a) is $t \vee x$, and if not we can finish using (a) and an easy induction on the length of the shortest maximal chain from $b$ to $b \vee x$. The proof that $b \wedge x \equiv t \wedge x$ is dual, using condition (b). Thus $\Theta$ is a congruence.

Define a map $\eta: L \rightarrow(L / \Theta)[C]$. If $\{x\}$ is an equivalence class in $\Theta$ then map $x$ to $(\{x\}, 2)$ if $x>t$ for some $b<t$ with $b \equiv t$. Otherwise, map $x$ to $(\{x\}, 1)$. If $b<t$ with $b \equiv t$ then map $b$ to $(\{b, t\}, 1)$ and map $t$ to $(\{b, t\}, 2)$. By condition (a) and an inductive argument similar to the previous paragraph, if $\{x\}$ is an equivalence class, and $x>t$ for some $b \equiv t$, then $x>b$. Thus $x \geq t$ in $L$ if and only if $\{x\} \geq\{b, t\}$ in $L / \Theta$. From here it is easy to check that $\eta$ is an isomorphism.

We now restate and prove Theorem 4.

Theorem 4. A lattice $L$ is congruence normal if and only if there exists a $C N$ labeling $\gamma: E(L) \rightarrow P$ for some poset $P$.

Proof. First suppose that $L$ is congruence normal. We will show that $\mathrm{Cg}: E(L) \rightarrow$ $\operatorname{Irr}(\operatorname{Con}(L))$ is a CN-labeling. Let $C_{1}$ and $C_{2}$ be a fundamental pair. If $x \equiv a_{1}$ in some congruence on $L$, then $a_{2} \vee x \equiv a_{2} \vee a_{1}$, or in other words, $a_{2} \equiv y$, and therefore $b_{1} \wedge a_{2} \equiv b_{1} \wedge y$, or in other words, $x \equiv b_{1}$. Thus for any edge $(p, q)$ in $\left(E\left(C_{1}\right) \cup E\left(C_{2}\right)\right)-\left\{\left(x, a_{2}\right),\left(b_{1}, y\right)\right\}$, we have $p \equiv q$. We have shown that 
$\operatorname{Cg}\left(x, a_{1}\right) \geq \operatorname{Cg}(e)$ for any $e \in\left(E\left(C_{1}\right) \cup E\left(C_{2}\right)\right)-\left\{\left(x, a_{2}\right),\left(b_{1}, y\right)\right\}$ and in particular, $\operatorname{Cg}\left(x, a_{1}\right) \geq \operatorname{Cg}\left(b_{2}, y\right)$. If $b_{2} \equiv y$ in some congruence, then $b_{2} \wedge a_{1} \equiv y \wedge a_{1}$, or in other words $x \equiv a_{1}$. Thus $\operatorname{Cg}\left(x, a_{1}\right)=\mathrm{Cg}\left(b_{2}, y\right)$, which is condition (i). For condition (ii), we have established the first inequality, and also that $\operatorname{Cg}\left(b_{2}, y\right) \geq \operatorname{Cg}(e)$ for any $e \in E\left(C_{2}\right)-\left\{\left(x, a_{2}\right),\left(b_{2}, y\right)\right\}$. The second inequality of (ii) follows by the same argument with $C_{1}$ and $C_{2}$ switched. To prove that $\mathrm{Cg}$ is a CN-labeling, it remains to show that the labels on $C_{1}$ are all distinct. But since $C_{1}$ is a chain, if any two edges in $C_{1}$ have the same label, there is a contradiction to Proposition 21.

Conversely, suppose that $L$ has a CN-labeling $\gamma: E(L) \rightarrow P$ and without loss of generality, let $\gamma$ be surjective. Let $m$ be any minimal element of $P$ and let $\Theta_{m}$ be the equivalence relation generated by setting $x \equiv a$ for any edge $(x, a)$ with label $m$. We will show that $\Theta_{m}$ is an edge contraction satisfying the conditions of Proposition 23. First, suppose some equivalence class $E$ has cardinality larger than two. Then either $E$ contains three elements $f<g<h$, or three distinct elements $a_{1}>x<a_{2}$. It is easy to check that when $L$ itself is not a chain, then any three elements $f<g<h$ are contained in the same chain in some fundamental pair, so the fact that $\gamma(f, g)=\gamma(g, h)=m$ contradicts condition (iii) for the CNlabeling $\gamma$. If $E$ contains distinct elements $a_{1}>x<a_{2}$ then let $y:=a_{1} \vee a_{2}$ and choose a fundamental pair of chains in $[x, y]$. Then by condition (i) of a CN-labeling $\gamma\left(b_{1}, y\right)=\gamma\left(x, a_{2}\right)$, but $\gamma\left(a_{1}, x\right)=\gamma\left(a_{2}, x\right)=m$, again contradicting condition (iii). Thus each equivalence class in $\Theta$ has cardinality 1 or 2 .

Suppose $b \equiv t$ for some $b<t$ and $b^{\prime}>b$ for some $b^{\prime} \neq t$, and consider the fundamental interval $\left[b, t \vee b^{\prime}\right]$. Now $t \vee b^{\prime}>t$ and $t \vee b^{\prime}>b^{\prime}$, because otherwise there would exist some fundamental pairs such that one of the chains would have more than two edges, and in that case, conditions (ii) and (iii) on $\gamma$ would contradict the minimality of $m$ in $P$. Thus by condition (i), $\gamma\left(b^{\prime}, t \vee b^{\prime}\right)=\gamma(b, t)$ so $b^{\prime} \equiv t \vee b^{\prime}$. We have shown that $t \vee b^{\prime}$ is the element $t^{\prime}$ required in condition (a) of Proposition 23. Condition (b) is verified by the dual argument. By Proposition 23, $L$ is obtained from $L / \Theta_{m}$ by doubling, and by Proposition $22, L / \Theta_{m}$ inherits a CN-labeling. By induction, $L / \Theta_{m}$ is congruence normal, and the proof is complete.

The proof of Theorem 4 leads to several insights about CN-labelings. First of all, for any order ideal $I$ in $P$ there is a congruence $\Theta_{I}$, generated by identifying the elements of all pairs with labels in $I$. By the same token, an order filter yields a quotient of $L$. A linear extension of $P$ encodes a sequence in which $L$ can be reduced to a one-element lattice by quotients, each of which reverses a doubling. The poset $P$ of labels can always be taken to be $\operatorname{Irr}(\operatorname{Con}(L))$, the poset of join-irreducibles of the congruence lattice of $L$. In fact, the labeling $\operatorname{Cg}: E(L) \rightarrow \operatorname{IrrCon}(L)$ is universal in the sense of the following proposition, which can be proven by an induction similar to the proof of Theorem 4. 
Proposition 24. Let $L$ be a lattice with a $C N$-labeling $\gamma: E(L) \rightarrow P$. Then there is an order-preserving map $f: \operatorname{Irr}(\operatorname{Con}(L)) \rightarrow P$ such that $\gamma=f \circ \mathrm{Cg}$.

\section{Simplicial arrangements and congruence uniformity}

In this section we apply Theorem 4 to posets of regions of simplicial hyperplane arrangements. Specifically, we obtain Theorem 25, a simple way to determine if a simplicial hyperplane arrangement is congruence uniform, and Theorem 26 , a simple way to determine if a simplicial hyperplane arrangement has a quotient ordering. (Recall that a lattice is congruence uniform if and only if it is semi-distributive and congruence normal.) In connection with Theorem 25, we cut the hyperplanes in a simplicial arrangement into pieces which we call shards. When $\mathcal{A}$ is simplicial, the shards correspond to join-irreducible congruences of $\mathcal{P}(\mathcal{A}, B)$ and we define a directed graph on the shards whose transitive closure is $\operatorname{Irr}(\operatorname{Con}(\mathcal{P}(A, B)))$. We give an example of a simplicial arrangement $\mathcal{A}$ and base region $B$ such that $\mathcal{P}(\mathcal{A}, B)$ is not congruence uniform. The section ends with a short proof (Proposition 28) that Coxeter arrangements satisfy the hypotheses of Theorem 26. This, combined with Theorem 3, accomplishes a simple proof of Theorem 27, which states that the weak order on a finite Coxeter group is a congruence uniform lattice. The proof given in [4] actually establishes a stronger property of the weak order, the $\mathcal{H} \mathcal{H}$ property. The present proof relies on Theorem 4, which is inspired by the ideas in [4].

We now define two directed graphs which are used to characterize quotient orders and congruence normality in the simplicial case. Let $\mathcal{A}$ be an arrangement with base region $B$, and let $\mathcal{A}^{\prime}$ be a rank-two subarrangement. In other words $\mathcal{A}^{\prime}$ consists of all the hyperplanes containing some subspace $L$ of codimension 2 , and $|\mathcal{A}| \geq 2$. There is a unique region $B^{\prime}$ of $\mathcal{A}^{\prime}$ containing $B$, and the hyperplanes in $\mathcal{A}^{\prime}$ bounding $B^{\prime}$ will be called basic hyperplanes in $\mathcal{A}^{\prime}$. Define a directed graph $\mathcal{Q}(\mathcal{A}, B)$ on the hyperplanes in $\mathcal{A}$ by letting $H \rightarrow H^{\prime}$ whenever $H$ is a basic hyperplane in some ranktwo subarrangement and $H^{\prime}$ is a non-basic hyperplane in the same subarrangement. To define the second directed graph we need to decompose the hyperplanes in $\mathcal{A}$ into shards. For each non-basic $H \in \mathcal{A}^{\prime}$, cut $H$ into two connected components by removing $L$ from $H$. Do this cutting for each rank-two subarrangement, and call the resulting connected components of the hyperplanes shards. Define $\operatorname{Sh}(\mathcal{A}, B)$, the directed graph whose vertices are the shards, and whose arrows are as follows: For each rank-two subarrangement, with $L$ as above, there is a directed arrow $\Sigma_{1} \rightarrow \Sigma_{2}$ whenever $\Sigma_{1}$ intersects $L$ and $\Sigma_{2}$ is incident to but does not intersect $L$. In particular, $\Sigma_{1}$ is contained in a basic hyperplane of the subarrangement and $\Sigma_{2}$ is in a non-basic hyperplane of the subarrangement. 
Theorem 25. Given a simplicial arrangement $\mathcal{A}$, the lattice $\mathcal{P}(\mathcal{A}, B)$ is congruence uniform if and only if $\mathcal{S h}(\mathcal{A}, B)$ is acyclic, in which case the transitive closure of $\mathcal{S h}(\mathcal{A}, B)$ is isomorphic to $\operatorname{Irr}(\operatorname{Con}(\mathcal{P}(\mathcal{A}, B)))$.

Proof. The graph $\mathcal{S} h(\mathcal{A}, B)$ can be defined on any hyperplane arrangement. So, for a moment let $\mathcal{A}$ be an arbitrary hyperplane arrangement and let $\gamma: E(\mathcal{P}(\mathcal{A}, B)) \rightarrow$ $\mathcal{A}$ label each edge $R_{1} \lessdot R_{2}$ in $\mathcal{P}(\mathcal{A}, B)$ by the shard containing the interior of the boundary of $R_{1} \cap R_{2}$. Let $C_{1}$ and $C_{2}$ be a fundamental pair of chains with $x, y, a_{1}$, $a_{2}, b_{1}$ and $b_{2}$ as usual. It is known [9] that any maximal chain in $\mathcal{P}(\mathcal{A}, B)$ crosses each hyperplane exactly once. Since the shards are components of the hyperplanes the labels on the edges of $C_{1}$ are all distinct. If in addition $\mathcal{A}$ is simplicial, any two hyperplanes bounding the region $x$ intersect in a codimension two subspace. Thus the separating set $S(x, y)$ is a rank-two subarrangement and $\gamma\left(x, a_{1}\right)=\gamma\left(b_{2}, y\right)$, because $\gamma\left(x, a_{1}\right)$ is a shard in a basic hyperplane of the subarrangement. If $\operatorname{Sh}(\mathcal{A}, B)$ is acyclic, then the transitive closure $\overline{\mathcal{S h}}(\mathcal{A}, B)$ is a partial order on $\mathcal{A}$. Condition (ii) for a CN-labeling is satisfied by definition of $\overline{\mathcal{S h}}(\mathcal{A}, B)$. So $\mathcal{P}(\mathcal{A}, B)$ is congruence normal, and thus congruence uniform by Theorem 3 .

For each shard $\Sigma$, define $\Phi(\Sigma)$ to be the smallest congruence with $R_{1} \equiv R_{2}$ whenever $R_{1}<R_{2}$ and the relative interior of $R_{1} \cap R_{2}$ is contained in $\Sigma$. To show that $\Phi(\Sigma)$ is join-irreducible, suppose $R_{1}, R_{2}, Q_{1}$ and $Q_{2}$ are regions such that $R_{1} \lessdot R_{2}, Q_{1}<Q_{2}$. Furthermore, suppose $R:=R_{1} \cap R_{2}$ and $Q:=Q_{1} \cap Q_{2}$ have interiors contained in $\Sigma$, and suppose $R \cap Q$ has codimension two. If $R_{1} \leq Q_{1}$ then, since $Q_{1} \nsucceq R_{2}$, in any congruence we have $R_{1} \equiv R_{2}$ if and only if $Q_{1} \equiv Q_{2}$. The same is true if $R_{1} \geq Q_{1}$. If $R_{1}$ and $Q_{1}$ are incomparable, then there are at least two hyperplanes containing $R \cap Q$ but not containing $\Sigma$, with the base region lying between two of the hyperplanes. This contradicts the hypothesis that the interiors of $R$ and $Q$ are contained in the same shard $\Sigma$. We have shown that if a congruence has $R_{1} \equiv R_{2}$ for some regions $R_{1}<R_{2}$ with the interior of $R_{1} \cap R_{2}$ contained in $\Sigma$, then that congruence contains $\Phi(\Sigma)$. So $\Phi(\Sigma)$ is $\operatorname{Cg}\left(R_{1}, R_{2}\right)$ for any such $R_{1}$ and $R_{2}$, and in particular is join-irreducible. Since every edge in $\mathcal{P}(\mathcal{A}, B)$ is contained in some shard, $\Phi$ is a surjective map to $\operatorname{Irr}(\operatorname{Con}(\mathcal{P}(\mathcal{A}, B)))$.

If $\Sigma_{1} \rightarrow \Sigma_{2}$ in $\mathcal{S} h(\mathcal{A}, B)$, then consideration of the regions incident to $L$ gives $\Phi\left(\Sigma_{1}\right) \geq \Phi\left(\Sigma_{2}\right)$. Thus $\Phi$ is an order-preserving surjection of the transitive closure of $\operatorname{Sh}(\mathcal{A}, B)$ onto $\operatorname{Irr}(\operatorname{Con}(\mathcal{P}(\mathcal{A}, B)))$. When $\mathcal{S h}(\mathcal{A}, B)$ is acyclic, we can let its transitive closure be the poset $P$ in Proposition 24. The order-preserving map $f$ found in Proposition 24 is surjective, and thus bijective, since a surjection $\Phi$ exists in the opposite direction. In particular, $\Phi$ is an order isomorphism whose inverse is $f$.

Conversely, suppose $\mathcal{A}$ is congruence uniform. Then in particular, the map $\mathrm{Cg}$ is a CN-labeling. As noted above, $\mathrm{Cg}$ is constant on shards and $\operatorname{Cg}\left(\Sigma_{1}\right)>\operatorname{Cg}\left(\Sigma_{2}\right)$ 
whenever $\Sigma_{1} \rightarrow \Sigma_{2}$. Thus $\mathcal{S h}(\mathcal{A}, B)$ is acyclic.

The following theorem can be proven using a similar but simpler argument. We omit the details.

Theorem 26. A simplicial arrangement $\mathcal{A}$ has a quotient ordering with respect to $B$ if and only if $\mathcal{Q}(\mathcal{A}, B)$ is acyclic, in which case any linear extension of the transitive closure of $\mathcal{Q}(\mathcal{A}, B)$ is a quotient ordering.

Figure 5 shows a simplicial hyperplane arrangement $\mathcal{A}$ with base region $B$ such that $\mathcal{P}(\mathcal{A}, B)$ is not congruence uniform. Some of the shards are shaded and labeled, and

$$
\Sigma_{1} \rightarrow \Sigma_{2} \rightarrow \Sigma_{3} \rightarrow \Sigma_{4} \rightarrow \Sigma_{1}
$$

is a cycle in $\mathcal{S} h(\mathcal{A}, B)$. The example in Figure 5 comes from Grünbaum's list of simplicial line arrangements in the projective plane [14]. We are not aware of an example where $\operatorname{Sh}(\mathcal{A}, B)$ is acyclic but $\mathcal{Q}(\mathcal{A}, B)$ is not, but we expect that one exists.
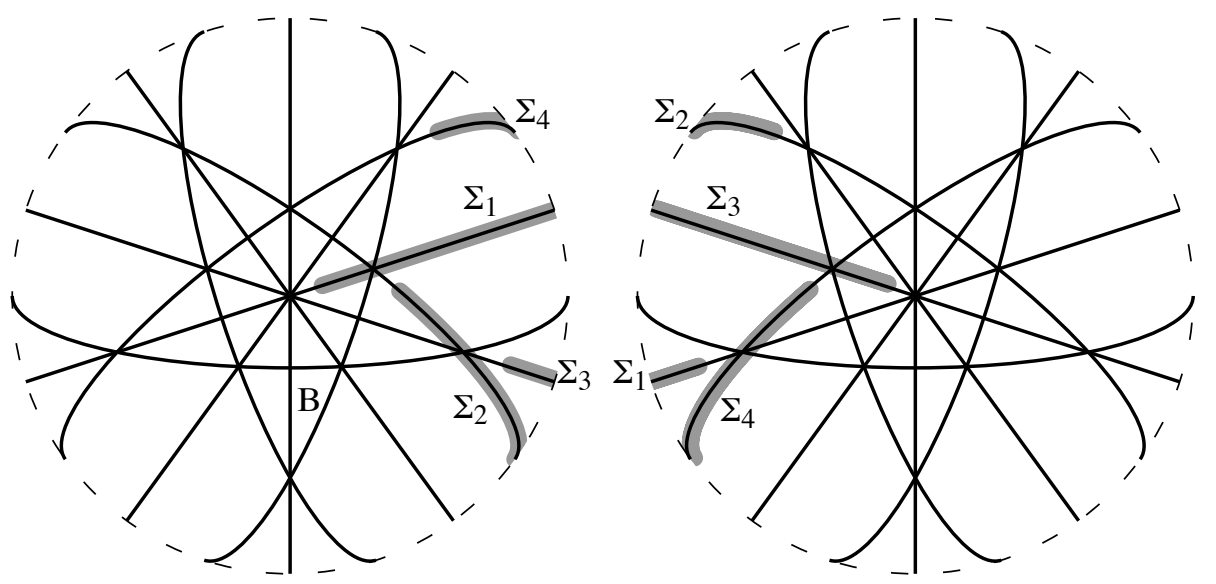

Figure 5. A simplicial arrangement $\mathcal{A}$ with base region $B$ such that $\mathcal{P}(\mathcal{A}, B)$ is not congruence uniform. The shards $\Sigma_{1}, \Sigma_{2}, \Sigma_{3}$ and $\Sigma_{4}$ form a cycle in $\mathcal{S h}(\mathcal{A}, B)$.

Theorem 26 can be used to prove that the poset of regions of a Coxeter arrangement has a quotient ordering, which in turn proves the following theorem.

Theorem 27 ([4]). The weak order on a finite Coxeter group is a congruence uniform lattice. 
In particular, the weak order on a finite Coxeter group is congruence normal and, in light of Theorem 3, congruence uniform. There are several ways to see that $\mathcal{Q}(\mathcal{A}, B)$ is acyclic when $\mathcal{A}$ is a Coxeter arrangement. In [4], Caspard, Le Conte de Poly-Barbut and Morvan used an edge-labeling scheme to prove that weak orders have the $\mathcal{H} \mathcal{H}$ property, a stronger property than congruence uniformity. Part of their proof, translated into the language of this paper, was to verify that if $H \rightarrow H^{\prime}$ then $l(H)<l\left(H^{\prime}\right)$. Here $l(H)$ is the Coxeter group length of the group element corresponding to reflection through $H$. In particular, $\mathcal{Q}(\mathcal{A}, B)$ is acyclic.

Since this proof uses Coxeter group methods which have not been explained here, we give a short elementary proof. The angle between a vector $v$ and a hyperplane $H$ is the minimum over vectors $x \in H$ of the angle between $x$ and $v$, denoted $\angle(v, H)$. A simplicial arrangement $\mathcal{A}$ is called equiangular if, whenever two or more hyperplanes intersect in a codimension three subspace $L$, the hyperplanes intersecting in $L$ can be ordered $H_{1}, H_{2}, \ldots H_{k}$ such that the angle between $H_{i}$ and $H_{i+1}$ is $2 \pi / k$. Coxeter arrangements are equiangular, and that fact can be exploited to prove that $\mathcal{Q}(\mathcal{A}, B)$ is acyclic.

Proposition 28. If $\mathcal{A}$ is a finite Coxeter arrangement and $B$ is any region, then $\mathcal{Q}(\mathcal{A}, B)$ is acyclic.

Proof. Let $b$ be a unit vector in the interior of $B$. We will show that whenever $H_{1} \rightarrow H^{\prime}$ in $\mathcal{Q}(\mathcal{A}, B)$, we have $\angle\left(b, H_{1}\right)<\angle\left(b, H^{\prime}\right)$.

Suppose that $H_{1} \rightarrow H^{\prime}$ in $\mathcal{Q}(\mathcal{A}, B)$. Then $H_{1}$ is a basic hyperplane in some rank-two subarrangement containing $H^{\prime}$. Call the other basic hyperplane $H_{2}$. Let $v^{\prime}$ be a unit vector in $H^{\prime}$ such that $\angle\left(b, H^{\prime}\right)=\angle\left(b, v^{\prime}\right)$. Let $l$ be the shortest path on the unit sphere from $b$ to $v^{\prime}$. There is a unique shortest path because $b$ is not antipodal to $v^{\prime}$. If $l$ intersects $H_{1}$ in some vector $v_{1}$ then since $H_{1} \neq H^{\prime}$, we have

$$
\angle\left(b, H^{\prime}\right)=\angle\left(b, v^{\prime}\right)>\angle\left(b, v_{1}\right) \geq \angle\left(b, H_{1}\right) .
$$

Otherwise, $l$ intersects $H_{2}$ in some vector $v_{2}$. Let $l^{\prime}$ be the reflection of $l$ in the hyperplane $H_{2}$. Then $l^{\prime}$ intersects $H_{1}$ in some vector $v_{1}$ with $\angle\left(v_{2}, v_{1}\right) \leq \angle\left(v_{2}, v^{\prime}\right)$. The angle between vectors is a metric on the sphere, so by the triangle inequality,

$$
\angle\left(b, H^{\prime}\right)=\angle\left(b, v_{2}\right)+\angle\left(v_{2}, v^{\prime}\right) \geq \angle\left(b, v_{2}\right)+\angle\left(v_{2}, v_{1}\right) \geq \angle\left(b, v_{1}\right) \geq \angle\left(b, H_{1}\right) .
$$

Equality would imply in particular that a shortest path from $v_{1}$ to $v^{\prime}$ would go through $v_{2}$, so we have $\angle\left(b, H^{\prime}\right)>\angle\left(b, H_{1}\right)$.

\section{Acknowledgments}

The author wishes to thank his advisor, Vic Reiner, as well as Kyle Calderhead and John Stembridge for helpful conversations. 


\section{REFERENCES}

[1] H. Barcelo and E. Ihrig, Modular elements in the lattice $L(A)$ when $A$ is a real reflection arrangement, Selected papers in honor of Adriano Garsia, Discrete Math. 193 (1998), 61-68.

[2] A. Björner, P. Edelman and G. Ziegler, Hyperplane Arrangements with a Lattice of Regions, Discrete Comput. Geom. 5 (1990), 263-288.

[3] N. Caspard, The lattice of permutations is bounded, Internat. J. Algebra Comput 10 (2000), 481-489.

[4] N. Caspard, C. Le Conte de Poly-Barbut and M. Morvan, Cayley lattices of finite Coxeter groups are bounded, Adv. in Appl. Math., to appear.

[5] I. Chajda and V. Snášel, Congruences in Ordered Sets, Math. Bohem. 123 (1998), 95-100.

[6] A. Day, Splitting lattices generate all lattices, Algebra Universalis 7 (1977), 163-169.

[7] A. Day, Congruence normality: the characterization of the doubling class of convex sets, Algebra Universalis 31 (1994), 397-406.

[8] V. Duquenne and A. Cherfouh, On Permutation Lattices, Math. Social Sci. 27 (1994), 73-89.

[9] P. Edelman, A Partial Order on the Regions of $\mathbb{R}^{n}$ Dissected by Hyperplanes, Trans. Amer. Math. Soc. 283, (1984), 617-631.

[10] P. Edelman and R. Jamison, The theory of convex geometries, Geom. Dedicata 19 (1985), $247-270$.

[11] S. Flath, The order dimension of multinomial lattices, Order 10 (1993), 201-219.

[12] R. Freese, J. Ježek and J. Nation, Free lattices, Mathematical Surveys and Monographs, 42, American Mathematical Society, 1995.

[13] G. Grätzer, General Lattice Theory, Second edition, Birkhäuser Verlag, Basel, 1998.

[14] B. Grünbaum, Arrangements of hyperplanes, Proceedings of the Second Louisiana Conference on Combinatorics, Graph Theory and Computing, 41-106. Louisiana State Univ., Baton Rouge, La., 1971.

[15] J. Humphreys, Reflection Groups and Coxeter Groups, Cambridge Studies in Advanced Mathematics, 29, Cambridge Univ. Press, 1990.

[16] M. Jambu and L. Paris, Combinatorics of Inductively Factored Arrangements, European J. Combin. 16 (1995), 267-292.

[17] C. Le Conte de Poly-Barbut, Sur les Treillis de Coxeter Finis (French), Math. Inf. Sci. hum. 32, 125 (1994), 41-57.

[18] R. McKenzie, Equational bases and nonmodular lattice varieties, Trans. Amer. Math. Soc. 174 (1972), 1-43.

[19] P. Orlik and H. Terao, Arrangements of hyperplanes, Grundlehren der Mathematischen Wissenschaften [Fundamental Principles of Mathematical Sciences], 300. Springer-Verlag, Berlin, 1992.

[20] I. Rabinovitch and I. Rival, The Rank of a Distributive Lattice, Discrete Math. 25 (1979), $275-279$.

[21] N. Reading, Order dimension, strong Bruhat order and lattice properties for posets, Order 19 (2002), 73-100.

[22] N. Reading, Lattice congruences of the weak order, in preparation.

[23] W. Trotter, Combinatorics and Partially Ordered Sets: Dimension Theory, Johns Hopkins Series in the Mathematical Sciences, The Johns Hopkins Univ. Press, 1992.

[24] G. Ziegler, Combinatorial construction of logarithmic differential forms, Adv. Math. 76 (1989), 116-154.

NATHAN READING

Mathematics Department, University of Michigan, Ann Arbor, MI 48109-1109, USA

e-mail:nreading@umich.edu

$U R L:$ http://www.math.lsa.umich.edu/ nreading/ 